\title{
Aspects of the conjugacy class structure of simple algebraic groups
}

\author{
Martin Cook
}

Received: 11 July 2006 / Accepted: 2 June 2009 / Published online: 26 June 2009

(C) Springer Science+Business Media, LLC 2009

\begin{abstract}
Let $G$ be an adjoint simple algebraic group over an algebraically closed field of characteristic $p$; let $\Phi$ be the root system of $G$, and take $t \in \mathbb{N}$. Lawther has proven that the dimension of the set $G_{[t]}=\left\{g \in G: g^{t}=1\right\}$ depends only on $\Phi$ and $t$. In particular the value is independent of the characteristic $p$; this was observed for $t$ small and prime by Liebeck. Since $G_{[t]}$ is clearly a disjoint union of conjugacy classes the question arises as to whether a similar result holds if we replace $G_{[t]}$ by one of those classes. This paper provides a partial answer to that question. A special case of what we have proven is the following. Take $p, q$ to be distinct primes and $G_{p}$ and $G_{q}$ to be adjoint simple algebraic groups with the same root system and over algebraically closed fields of characteristic $p$ and $q$ respectively. If $s \in G_{p}$ has order $q$ then there exists an element $u \in G_{q}$ such that $o(u)=o(s)$ and $\operatorname{dim} u^{G_{q}}=\operatorname{dim} s^{G_{p}}$.
\end{abstract}

Keywords Algebraic groups · Conjugacy classes · Characteristic independent

\section{Background and main results}

The present paper comes from the $\mathrm{PhD}$ thesis of the author; the main results of which are given below. However first we must make some definitions. So let $X$ and $Y$ be reductive groups (which for us means they are connected) over algebraically closed fields with (possibly different) prime characteristics. A reductive group $X$ is said to have type $(\Psi, n)$ if $\operatorname{rank} X=n$ and the commutator subgroup, $[X, X]$, is semisimple with root system $\Psi$. If $X$ is simple we will often abuse this notation and refer to $\Psi$ as the type; furthermore if $\Lambda$ is a family of irreducible root systems and $X$ is simple, we say $X$ has type $\Lambda$ if $\Psi \in \Lambda$. We write $X \sim_{t} Y$ if $X$ and $Y$ have the same type;

The author gratefully acknowledges the support of the EPSRC during the preparation of this work.

M. Cook (凶)

Department of Mathematics and Statistics, Lancaster University, Lancaster, LA1 4YF, UK

e-mail:m.cook@lancaster.ac.uk 
this is clearly an equivalence relation. Next let $\Phi$ be a root system, $p$ a prime and let $G(\Phi)$ be an adjoint simple algebraic group of type $\Phi$ (we write $G(\Phi)_{p}$ if the group is over $\overline{\mathbb{F}_{p}}$ ). It is worth stating at this point that throughout this paper all groups will be over algebraically closed fields (in fact all fields are of the form $\overline{\mathbb{F}_{p}}$ ); we are not considering rationality properties here. Now set

$$
\begin{array}{r}
\Pi(X, \Phi)=\left\{s: s \text { is a semisimple element of the group } G(\Phi)_{p},\right. \\
\text { for some prime } \left.p, \text { and } C_{G(\Phi)_{p}}(s)^{\circ} \sim_{t} X\right\} .
\end{array}
$$

(Recall that since $s$ is a semisimple element, $C_{G(\Phi)_{p}}(s)^{\circ}$ is reductive.) Next set

$$
o_{\min }(X, \Phi)=\min \{o(s): s \in \Pi(X, \Phi)\}
$$

(here $o(s)$ is the order of the group element $s$ ) and for a prime number $q$ set

$$
q^{(X, \Phi)}=\min \left\{q^{e}: q^{e} \geq o_{\min }(X, \Phi)\right\} .
$$

Now given a reductive subgroup $X \leq G(\Phi)_{p}$ we say that a prime $q$ is admissible with respect to $X$ if for some prime $r$ and nonnegative integer $i$ there is a semisimple element $s \in G(\Phi)_{r}$ such that $o(s)=q^{i}$ and $C_{G(\Phi)_{r}}(s)^{\circ} \sim_{t} X$. Note that the identity element is semisimple and of order $1=q^{0}$. Thus every prime $q$ is admissible with respect to $X=G(\Phi)_{p} \sim_{t} G(\Phi)_{r}=C_{G(\Phi)_{r}}(1)^{\circ}$. Finally we come to the main results.

Theorem 1 Let $G_{p}=G(\Phi)_{p}$ be an adjoint simple algebraic group of type $\Phi$ over $\overline{\mathbb{F}_{p}}$. Let $X \leq G_{p}$ be the connected centralizer of a semisimple element; if $\Phi=B_{n}, C_{n}$ or $D_{n}$ for some $n$ let $q$ be admissible with respect to $X$, otherwise let $q$ be any prime. Then there exists a unipotent conjugacy class $\mathcal{C} \subset G_{q}=G(\Phi)_{q}$ such that for any $u \in \mathcal{C}$

$$
\operatorname{dim} C_{G_{q}}(u)=\operatorname{dim} X \quad \text { and } \quad q^{(X, \Phi)}=o(u) \text { or } q . o(u) .
$$

Equality between $q^{(X, \Phi)}$ and $o(u)$ holds in most cases and holds in all but one case if $q$ is a good prime. Full details are given in Theorem 4 at the end of the paper. There is an immediate corollary to Theorem 1.

Corollary 2 Given a semisimple element $s \in G_{p}=G(\Phi)_{p}$ and a prime $q$ which is admissible with respect to $X=C_{G_{p}}(s)^{\circ}$ if $\Phi=B_{n}, C_{n}$ or $D_{n}$ for some $n$, there is a unipotent element $u \in G_{q}=G(\Phi)_{q}$ such that $\operatorname{dim} C_{G_{q}}(u)=\operatorname{dim} X$ and $o(u)$ divides $\min \left\{q^{e}: q^{e} \geq o(s)\right\}$.

Proof Take any $u$ in the class $\mathcal{C}$ given by Theorem 1 and observe that $q^{(X, \Phi)}$ divides $\min \left\{q^{e}: q^{e} \geq o(s)\right\}$, since $o(s) \geq o$ min $(X, \Phi)$.

If we have a semisimple element of prime order we can make a stronger statement.

Theorem 3 Let $p, q$ be distinct primes and let $G_{p}$ and $G_{q}$ be as above. If $s \in G_{p}$ has order $q$ then there exists an element $u \in G_{q}$ such that $o(u)=o(s)$ and $\operatorname{dim} u^{G_{q}}=$ $\operatorname{dim} s^{G_{p}}$. 
In [5] it was proven that given a natural number $t$ and an adjoint simple algebraic group $G$ over an algebraically closed field of characteristic $p$, the dimension of the set $G_{[t]}=\left\{g \in G: g^{t}=1\right\}$ is independent of $p$. Clearly $G_{[t]}$ is a union of conjugacy classes. The author's thesis was an attempt to see what could be said if $G_{[t]}$ was replaced by one of those classes. The statement of Theorem 3 above is in the style of [5].

The proof of Theorem 1 will be given by case analysis in the following sections. The individual proofs are quite straightforward once certain functions are defined. These functions may appear to come from nowhere, but can be characterized combinatorially. This will be done in future papers and hints that a more uniform approach may be possible. For the moment though, this possibility has not been realized and we must stick with case analysis. So Section 2 recalls an important algorithm for finding all connected centralizers of semisimple elements in a simple algebraic group and uses this to prove Theorem 1 for the exceptional cases. Section 3 lays out our strategy for proving Theorem 1 . Sections 4 to 7 deal with types $A, C, D$ and $B$ respectively. Theorem 3 is proven in Section 8.

\section{Known results and exceptional groups}

This section recalls some well known results of Steinberg and a useful result from [2] and then applies them to the exceptional groups. Recall that if $s$ is a semisimple element, $T$ a maximal torus containing $s$ and $\left\{X_{\alpha}: \alpha \in \Phi\right\}$ the root subgroups with respect to $T$, then it can be shown that $C_{G}(s)^{\circ}=\left\langle T, X_{\alpha}: \alpha(s)=1\right\rangle$. Furthermore $C_{G}(s)^{\circ}$ is reductive with root system $\Psi=\{\alpha \in \Phi: \alpha(s)=1\}$. Finally if $G$ is simplyconnected then we have that $C_{G}(s)^{\circ}=C_{G}(s)$. This material is due to Steinberg and can be found in section 3.5 of [1]. Now given $t \in \mathbb{N}$ and a simple simply-connected group $G_{s c}$, let $G_{a d}$ be the adjoint group in the isogeny class of $G_{s c}$ and let $\phi: G_{s c} \rightarrow$ $G_{a d}$ be an isogeny. Also let $s \in G_{s c}$ be a semisimple element such that the order of $\phi(s)$ is $t$. Note that if $G_{a d}$ is over a field of characteristic $p$ then $p$ must not divide $t$. An algorithm is given in [2] for finding all possible centralizers of $s \in G_{s c}$. The following lemma is essentially taken from [5] and shows that the algorithm can actually be used to determine all possible connected centralizers of an element of order $t$ in $G_{a d}$.

Lemma 2.1 Let $\phi: G_{s c} \rightarrow G_{a d}$ be an isogeny and $s \in G_{s c}$ be a semisimple element then $\operatorname{dim} C_{G_{a d}}(\phi(s))=\operatorname{dim} C_{G_{s c}}(s)$ and $C_{G_{a d}}(\phi(s))^{\circ}=\phi\left(C_{G_{s c}}(s)\right)$.

Proof Suppose $c \in G_{s c}$ is such that $\phi(c) \in C_{G_{a d}}(\phi(s))$, then $c s c^{-1} s^{-1} \in \operatorname{Ker} \phi=$ $Z\left(G_{s c}\right)$. So let $z=c s c^{-1} s^{-1}$ and note that $c s c^{-1}=z s$. Now consider the set $G_{s c}(z, s)=\left\{c \in G_{s c}: c s c^{-1}=z s\right\}$ : we see that $c^{\prime} \in G_{s c}(z, s)$ implies $c s c^{-1}=$ $c^{\prime} s c^{\prime-1}$. This leads to $c^{\prime} \in c C_{G_{s c}}(s)$ and we see that $G_{s c}(z, s)$ is a coset of $C_{G_{s c}}(s)$. So $\operatorname{Ker} \phi$ being finite gives us $\operatorname{dim} \phi\left(C_{G_{s c}}(s)\right)=\operatorname{dim} C_{G_{s c}}(s)$ and since $G_{s c}(1, s)=$ $C_{G_{s c}}(s)$ we see that

$$
C_{G_{a d}}(\phi(s))=\phi\left(\bigcup_{z \in \operatorname{Ker} \phi} G_{s c}(z, s)\right)
$$


has $\phi\left(C_{G_{s c}}(s)\right)$ as a subgroup of finite index; therefore $\operatorname{dim} C_{G_{a d}}(\phi(s))=\operatorname{dim} C_{G_{s c}}(s)$ and $\phi\left(C_{G_{s c}}(s)\right)$ contains $C_{G_{a d}}(\phi(s))^{\circ}$. However $\phi\left(C_{G_{s c}}(s)\right)=\left\langle\phi(T), \phi\left(X_{\alpha}\right)\right.$ : $\alpha(s)=1\rangle$ is clearly contained in $C_{G_{a d}}(\phi(s))^{\circ}$ and so the lemma is proven.

We shall now give the algorithm as it is presented in [5]. If we assume that $G$ is adjoint it is not difficult to see that, given the above lemma, this statement is equivalent to that given in [2]. So let $\Phi$ be a root system of $G$ and $\Delta=\left\{\alpha_{1}, \ldots, \alpha_{n}\right\}$ a set of simple roots in $\Phi$. Let $\alpha_{0}$ be the highest root of $\Phi$ with respect to $\Delta$, set $c_{0}=1$ and define $c_{i}$ for $1 \leq i \leq n$ by $\alpha_{0}=\sum_{i=1}^{n} c_{i} \alpha_{i}$. The $c_{i}$ are called weights and can be used to label the extended Dynkin diagram of type $\widetilde{\Phi}$.

Algorithm 2.2 Using the notation above and taking any $t \in \mathbb{N}$ choose non-negative integers $b_{0}, b_{1}, \ldots, b_{n}$ with $\operatorname{gcd}\left(b_{0}, \ldots, b_{n}\right)=1$ satisfying $\sum_{i=0}^{n} b_{i} c_{i}=t$. The $b_{i}$ are called labels. Then the roots $\alpha_{i}$ (or $-\alpha_{0}$ if $\left.i=0\right)$ for which $b_{i}=0$ form a simple system $\Pi$ whose corresponding subsystem subgroup of $G$ (which I take to have maximal rank) is the connected centralizer of some semisimple element of order $t$. The Dynkin diagram of this connected centralizer is the subgraph of the extended Dynkin diagram spanned by those vertices with $b_{i}=0$. In fact up to conjugacy all connected centralizers of semisimple elements of order $t$ occur in this way.

From this we see that the Dynkin diagrams of connected centralizers of semisimple elements must be proper subgraphs of the extended Dynkin diagram of $G$. Also each proper subgraph of the extended Dynkin diagram of $\Phi$ will give a connected centralizer in some adjoint simple group of type $\Phi$. Simply let $b_{i}=1$ on each vertex you wish to delete and then choose $p$ coprime to $t=\sum_{i=0}^{n} b_{i} c_{i}$; the adjoint simple group of type $\Phi$ over $\overline{\mathbb{F}_{p}}$ will then contain a semisimple element of order $t$ whose connected centralizer has the required diagram. When thinking about connected centralizers from now on we will automatically consider the corresponding subgraph of the extended Dynkin diagram. Clearly we can use Algorithm 2.2 to list the $\sim_{t}$ classes of connected centralizers in groups of type $\Phi$; furthermore given an $X$ we can see all the possible orders of semisimple elements whose connected centralizer has the same type as $X$. This allows us to calculate the value of $o_{\min }(X, \Phi)$ and, given $q$, that of $q^{(X, \Phi)}$. We can demonstrate this process in the easiest of the exceptional cases $G_{2}$. We begin with the extended Dynkin diagram of type $\widetilde{G_{2}}$, together with the weights obtained from the highest root.

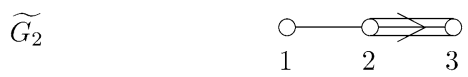

From this it is easy to see all possible proper subgraphs and the only task remaining is to find labels $b_{i}$ realizing each subgraph, satisfying $\operatorname{gcd}\left(b_{0}, \ldots, b_{n}\right)=1$ and minimizing $\sum_{i=0}^{n} b_{i} c_{i}$. The table below provides this information. Here $X_{1}^{a_{1}} \cdots X_{r}^{a_{r}} T_{l}$ will denote a reductive group with simple components of type $X_{1}, \ldots, X_{r}$ and multiplicities $a_{1}, \ldots, a_{r}$. The term $T_{l}$ is a central torus of rank $l$. For convenience we will sometimes have repeated simple components and sometimes write multiplicities, this should cause no confusion in context. We will also assume that $x_{1} \geq \cdots \geq x_{r}$, 
where $x_{i}=\operatorname{rank} X_{i}$. Note that from this representation of $X$ we can read off the rank $\left(a_{1} x_{1}+\cdots+a_{r} x_{r}+l\right)$, dimension (rank $X+|\Psi|$, where $\Psi$ is the root system of the semisimple group $[X, X]$ ) and (using Algorithm 2.2) the value of $q^{(X, \Phi)}$ for any prime $q$. Since these are the only properties we are interested in, we shall use this notation throughout the paper

\begin{tabular}{cccc}
\hline$X$ & $\operatorname{dim} X$ & Labels & $o_{\min }\left(X, G_{2}\right)$ \\
\hline$G_{2}$ & 14 & 100 & 1 \\
$A_{2}$ & 8 & 001 & 3 \\
$A_{1}^{2}$ & 6 & 010 & 2 \\
$A_{1} T_{1}$ & 4 & 110 & 3 \\
$T_{2}$ & 2 & 111 & 6 \\
\hline
\end{tabular}

We can now prove Theorem 1 in this case by explicitly giving unipotent conjugacy classes to pair with each $X$. The unipotent conjugacy classes in $G\left(G_{2}\right)_{q}$ are listed along with the dimensions of the corresponding centralizers in section 13.1 of [1]; the reader can check that the dimensions match. The Jordan structure of class representatives is given with respect to the module $V_{7}$ and is taken from [4]; it is easy to see that the order of each element in the class is the smallest power of $q$ greater than the size of the largest Jordan block. Note that we do not always have $q^{\left(X, G_{2}\right)}=o(u)$; the single exception is $2^{\left(A_{2}, G_{2}\right)}=2 . o(u)$.

\begin{tabular}{|c|c|c|c|c|c|}
\hline$X$ & $q$ & $q^{\left(X, G_{2}\right)}$ & $\begin{array}{c}\text { Class } \\
\mathcal{C} \subseteq G\left(G_{2}\right)_{q}\end{array}$ & $\begin{array}{c}\text { Jordan } \\
\text { Structure }\end{array}$ & $o(u)$ \\
\hline$G_{2}$ & $\geq 2$ & $q$ & 1 & $1^{14}$ & $q$ \\
\hline \multirow[t]{2}{*}{$A_{2}$} & 2 & 4 & $A_{1}$ & $2^{2} 1^{3}$ & 2 \\
\hline & $\geq 3$ & $q$ & & $2^{2} 1^{3}$ & $q$ \\
\hline$A_{1}^{2}$ & $\geq 2$ & $q$ & $\tilde{A_{1}}$ & $2^{3} 1$ & $q$ \\
\hline \multirow[t]{2}{*}{$A_{1} T_{1}$} & 2 & 4 & $G_{2}\left(a_{1}\right)$ & $3^{2} 1$ & 4 \\
\hline & $\geq 3$ & $q$ & & $3^{2} 1$ & $q$ \\
\hline \multirow[t]{4}{*}{$T_{2}$} & 2 & 8 & $G_{2}$ & 61 & 8 \\
\hline & 3 & 9 & & 7 & 9 \\
\hline & 5 & 25 & & 7 & 25 \\
\hline & $\geq 7$ & $q$ & & 7 & $q$ \\
\hline
\end{tabular}

We will not give full details of the other exceptional cases since the techniques are similar. For the case of $F_{4}$ we will give the extended Dynkin diagram, together with the weights obtained from the highest root, and the list of $\sim_{t}$ classes of connected centralizers of semisimple elements, together with the values of $o_{\min }\left(X, F_{4}\right)$. The reader can then verify Theorem 1 themselves using the information given in [1] and [4]. It will then be seen that as above we do not always have $q^{\left(X, F_{4}\right)}=o(u)$; the exceptions are $(X, q)=\left(A_{3} A_{1}, 3\right),\left(A_{3} T_{1}, 2\right),\left(A_{3} T_{1}, 5\right)$ and $\left(C_{2} T_{2}, 2\right)$. In these cases we can choose classes such that $q^{\left(X, F_{4}\right)}=q . o(u)$. 


\begin{tabular}{|c|c|c|c|}
\hline \multirow[t]{2}{*}{$\widetilde{F_{4}}$} & \multicolumn{3}{|c|}{$0-0-2>0-0$} \\
\hline & 1 & 2 & 2 \\
\hline$X$ & $\operatorname{dim} X$ & Labels & $o_{\min }\left(X, F_{4}\right)$ \\
\hline$F_{4}$ & 52 & 10000 & 1 \\
\hline$C_{3} A_{1}$ & 24 & 01000 & 2 \\
\hline$A_{2}^{2}$ & 16 & 00100 & 3 \\
\hline$A_{3} A_{1}$ & 18 & 00010 & 4 \\
\hline$B_{4}$ & 36 & 00001 & 2 \\
\hline$C_{3} T_{1}$ & 22 & 11000 & 3 \\
\hline$A_{2} A_{1} T_{1}$ & 12 & 10100 & 4 \\
\hline$B_{3} T_{1}$ & 22 & 10001 & 3 \\
\hline$A_{1}^{3} T_{1}$ & 10 & 01010 & 6 \\
\hline$C_{2} A_{1} T_{1}$ & 14 & 01001 & 4 \\
\hline$A_{3} T_{1}$ & 16 & 00011 & 6 \\
\hline$A_{2} T_{2}$ & 10 & 11100 & 6 \\
\hline$C_{2} T_{2}$ & 12 & 11001 & 5 \\
\hline$A_{1}^{2} T_{2}$ & 8 & 10101 & 6 \\
\hline$A_{1} T_{3}$ & 6 & 11101 & 8 \\
\hline$T_{4}$ & 4 & 11111 & 12 \\
\hline
\end{tabular}

The groups of type $E$ are dealt with similarly. Although here we find classes satisfying $q^{(X, \Phi)}=o(u)$ in all cases. We can now move on to the classical groups.

\section{Strategy}

In this section we define certain sets and some properties of the functions between them that will be used in the following sections to prove Theorem 1 . So let $\Lambda \in$ $\{A, B, C, D\}$, let $[X]_{t}$ denote the $\sim_{t}$ class of a reductive group $X$ and consider the following sets

$$
\begin{array}{r}
\operatorname{Cent}(\Lambda)=\left\{[X]_{t}: X=C_{G\left(\Lambda_{n}\right)_{p}}(s)^{\circ} \text { for some semisimple element } s \in G\left(\Lambda_{n}\right)_{p},\right. \\
\text { some } n \in \mathbb{N} \text { and some prime } p\} .
\end{array}
$$

To avoid possible confusion, we emphasize at this point that $\operatorname{Cent}(\Lambda)$ contains the $\sim_{t}$ classes of connected centralizers in any group of type $\Lambda$. Also to avoid confusion, we point out that we will often abuse notation and write ' $X \in \operatorname{Cent}(\Lambda)$ ' rather than ' $[X]_{t} \in \operatorname{Cent}(\Lambda)$ '. Furthermore when we write ' $\operatorname{dim} X$ ' (resp. ' $\operatorname{rank} X$ ') for $X \in \operatorname{Cent}(\Lambda)$, we mean the dimension (resp. rank) of any representative of $[X]_{t}$, since these are invariants of the class. Finally let

$$
\operatorname{Cent}_{q}(\Lambda)=\left\{[X]_{t} \in \operatorname{Cent}(\Lambda): q \text { is admissible with respect to } X\right\} .
$$

(Clearly if $q$ is admissible with respect to $X$ then it is admissible with respect to any group in the class $[X]_{t}$.) To illustrate these concepts we shall now calculate $\operatorname{Cent}(A)$. 
The extended Dynkin diagram of type $\widetilde{A_{n}}$, together with the weights obtained from the highest root is shown below.

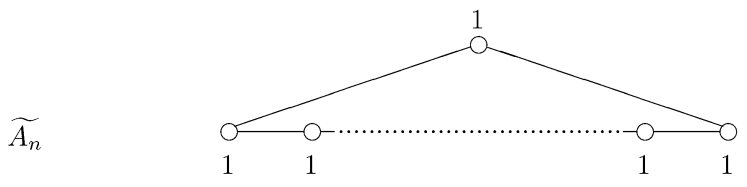

Clearly all proper subgraphs are unions of Dynkin diagrams of type $A$ and so Algorithm 2.2 shows us that all connected centralizers have the form $X=A_{n_{1}} \ldots A_{n_{r}} T_{l}$ (repeated factors are allowed). Also note that $l+1$ is the number of vertices removed (there are $\sum n_{i}+l+1$ vertices in the extended diagram and those not removed span the Dynkin diagram of $[X, X]$, which has rank $\left.\sum n_{i}\right)$ and since the diagram is a cycle this is no less than $r$, the number of connected components left after the removals. Finally the diagram tells us that every prime is admissible with respect to every connected centralizer. This follows since all weights are 1, so we can label $l$ of the vertices we remove in Algorithm 2.2 by a 1 and the last vertex by $q^{i}-l$, where $i$ is chosen to be large enough to make this positive. Thus $q$ may be chosen arbitrarily unless only one vertex is removed. In this case that vertex must be labeled with a 1 and the corresponding subsystem subgroup must be the connected centralizer of an element of order 1, i.e. it must be the whole group and again every prime is admissible. Thus all the sets $\operatorname{Cent}_{q}(A)$ are the same; they are all equal to $\operatorname{Cent}(A)$ which equals

$$
\left\{A_{n_{1}} \ldots A_{n_{r}} T_{l}: n_{1}, \ldots, n_{r}, l \in \mathbb{N} \cup\{0\}, \text { where } n_{1} \geq \cdots \geq n_{r} \text { and } l+1 \geq r\right\} .
$$

Next we return to $\Lambda \in\{A, B, C, D\}$ and consider the sets

$$
\operatorname{uccl}_{q}(\Lambda)=\left\{\left(\mathcal{C}, G\left(\Lambda_{n}\right)_{q}\right): n \in \mathbb{N} \text { and } \mathcal{C} \subseteq G\left(\Lambda_{n}\right)_{q} \text { is a unipotent conjugacy class }\right\}
$$

As with $\operatorname{Cent}(\Lambda)$ we try to avoid confusion by stating that this set is not the set of all unipotent classes in a single group. Now each pair $\mathbf{m}=\left(\mathcal{C}, G\left(\Lambda_{n}\right)_{q}\right) \in \operatorname{uccl}_{q}(\Lambda)$ has certain numbers associated to it, the simplest of which is the rank of the pair. This is denoted $r(\mathbf{m})$ and equals $n$. Before stating the others we set the convention that when we write ' $u \in \mathbf{m}$ ' we mean $u \in \mathcal{C}$. So if $u \in \mathbf{m}$ then we know that the dimension of $C_{G\left(\Lambda_{n}\right)_{q}}(u)$ and the order of $u$ are both independent of $u$ and hence invariants of $\mathbf{m}$. These will be called the centralizer dimension of $\mathbf{m}$ and the element order of $\mathbf{m}$ respectively. We will denote the centralizer dimension by c.dim $\mathbf{m}$ and the element order by $o_{e}(\mathbf{m})$. Finally we define a representation of $\operatorname{uccl}_{q}(\Lambda)$ to be a function from $\operatorname{uccl}_{q}(\Lambda)$ to $S$, where $S$ is a set whose elements we call symbols. Our results will be much more elegant if we use appropriate symbols to represent the elements of $\operatorname{uccl}_{q}(\Lambda)$ and we will generally identify those elements with their images in $S$.

The symbols in $S$ will be based on partitions of natural numbers, so before preceding we will now outline our conventions and notation regarding partitions. There appear to be various conventions in use, but the ones stated here will be used throughout this paper and in any sequels. We will always list the parts of a partition in descending order of size and unless multiplicities are explicitly given we will assume 
that parts may be repeated. So let $\mathbf{n}=\left(n_{1}, \ldots, n_{r}\right)$ be a partition and let $\alpha, \beta \in \mathbb{Z}$ then we write $\alpha \mathbf{n}+\beta=\left(\alpha n_{1}+\beta, \ldots, \alpha n_{r}+\beta\right)$ provided each $\alpha n_{i}+\beta \geq 0$. Also let $|\mathbf{n}|=\sum n_{i}$. Next consider $\mathbf{n}^{*}=\left(k_{1}, \ldots, k_{s}\right)$, where $k_{i}=\left|\left\{j: n_{j} \geq i\right\}\right|$. This is the dual partition of $\mathbf{n}$ and it is equal to $r^{n_{r}}(r-1)^{n_{r-1}-n_{r}} \cdots 2^{n_{2}-n_{3}} 1^{n_{1}-n_{2}}$; here the superscripts give the multiplicities of the parts they are above. We call the partition with no parts 'empty' and denote it 0 . By convention the dual of the empty partition is empty. If $\mathbf{m}=\left(m_{1}, \ldots, m_{z}\right)$ and $n_{r} \geq m_{1}$ then we can form the concatenation $(\mathbf{n}, \mathbf{m})=\left(n_{1}, \ldots, n_{r}, m_{1}, \ldots, m_{z}\right)$. We conclude our brief look at partitions with an easily verified lemma.

Lemma 3.1 If $\left(m_{1}, m_{2}, \ldots, m_{z}\right)^{*}=\mathbf{g}$ then $(\mathbf{g}-1)^{*}=\left(m_{2}, \ldots, m_{z}\right)$.

With everything we need about partitions in place we can turn to an example. We know from section 13.1 of [1] that the unipotent conjugacy classes of $G\left(A_{n}\right)_{q}$ are parameterized by the partitions of $n+1$ (the partitions are given by the block sizes of the Jordan form of any matrix in the class). Hence we will represent any $\mathbf{m} \in \operatorname{uccl}_{q}(A)$ by the partition given by the block sizes of the Jordan form of any matrix in the class determined by $\mathbf{m}$. The representation defined in this way is bijective and so we can identify the members of $\operatorname{uccl}_{q}(A)$ with the set $S=\{\mathbf{m}: \mathbf{m}$ is a partition of some $m \geq 2\}$. This is a good representation because key properties of the members of $\operatorname{uccl}_{q}(A)$ can be seen from the corresponding partition. It is obvious that $r(\mathbf{m})=$ $|\mathbf{m}|-1$, but section 13.1 of [1] also states that if $\mathbf{m}^{*}=\left(g_{1}, \ldots, g_{a}\right)$ then

$$
\text { c. } \operatorname{dim} \mathbf{m}=\sum_{i=1}^{a} g_{i}^{2}-1 .
$$

However the representation is not perfect; a small inconsistency arises as follows. For every prime $q$ there is a corresponding $\operatorname{set} \operatorname{uccl}_{q}(A)$ and a bijection between that set and $S$. Thus every member of $S$ is identified with infinitely many pairs and although we have seen above that all these pairs have the same rank and centralizer dimension they will not have the same element order. If $\mathbf{m} \in \operatorname{uccl}_{q}(A)$ is identified with the partition $\left(m_{1}, \ldots, m_{z}\right)$ then it is easily seen that $o_{e}(\mathbf{m})=\min \left\{q^{i}: q^{i} \geq m_{1}\right\}$. Thus we cannot determine the element order from the partition alone, but if we also know $q$ then we can. This shows us that when we identify $\operatorname{uccl}_{q}(A)$ with $S$ we are losing some information, but it shouldn't cause any practical problems since we will always specify which $\operatorname{uccl}_{q}(A)$ we are taking the partitions to be in when we talk about element orders.

With all our new sets now defined we can see that proving Theorem 1 for the classical groups is equivalent to constructing functions $f: \operatorname{Cent}_{q}(\Lambda) \rightarrow \operatorname{uccl}_{q}(\Lambda)$, for any $\Lambda \in\{A, B, C, D\}$ and any prime $q$, such that the following holds. For each $X \in \operatorname{Cent}_{q}(\Lambda)$ we have

(A) $\operatorname{rank} X=r(f(X))$;

(B) $\operatorname{dim} X=\mathrm{c} \cdot \operatorname{dim} f(X)$;

(C) $q^{\left(X, \Lambda_{n}\right)}=o_{e}(f(X))$ or $q . o_{e}(f(X))$, where $n=\operatorname{rank} X$.

Such functions will be explicitly given in the following sections. Sometimes a stronger version of property $(\mathrm{C})$ holds: namely that $q^{\left(X, \Lambda_{n}\right)}=o_{e}(f(X))$ for every 
$X \in \operatorname{Cent}_{q}(\Lambda)$. We call this property $(\mathrm{C}+)$. In future papers we will show that for each prime $q$ and $\Lambda \in\{A, B, C, D\}$ the functions we will give here are the unique functions between $\operatorname{Cent}_{q}(\Lambda)$ and $\operatorname{uccl}_{q}(\Lambda)$ which preserve certain natural partial orders and satisfy properties (A) and (B). Property (C) will thus be shown to be a consequence of order preservation and the other two properties. Why such functions, which we call sensible, should be unique or exist at all is still a mystery. It is also unclear why the poset structure, which appears to come from the representations, should have such a strong influence; uniqueness fails completely if we do not have a poset homomorphism.

\section{Type $A$}

We can now prove Theorem 1 for type $A$. We have already noted that for any prime $q$ the set $\operatorname{Cent}_{q}(A)$ is equal to $\operatorname{Cent}(A)$, so for each $q$ define a function $f_{A}$ : $\operatorname{Cent}(A) \rightarrow \operatorname{uccl}_{q}(A)$ as follows. Let $X=A_{n_{1}} \ldots A_{n_{r}} T_{l}$, where $n_{1} \geq \cdots \geq n_{r} \geq 1$, be a representative of some element in $\operatorname{Cent}(A)$ and let $\mathbf{m}=\left(n_{1}, \ldots, n_{r}\right)^{*}$, then set $f_{A}(X)=(l+1, \mathbf{m})$.

Theorem 4.1 The function $f_{A}$ is a bijection satisfying properties $(A),(B)$ and $(C+)$. Moreover if $p \neq q$ then there exists a semisimple element $s \in G\left(A_{n}\right)_{p}$ such that $C_{G\left(A_{n}\right)_{p}}(s) \sim_{t} X$ and $o(s)=q^{\left(X, A_{n}\right)}$.

Proof Recall from above that $l+1 \geq r$. Hence if $\left(m_{2}, \ldots, m_{z}\right)=\mathbf{m}=\left(n_{1}, \ldots, n_{r}\right)^{*}$ then $l+1 \geq r=m_{2}$ and so we can form the concatenation $(l+1, \mathbf{m})$, which is a partition of $n+1=\sum n_{i}+l+1$. Thus the map is well-defined and satisfies property (A). If $\mathcal{C}=\left(m_{1}, m_{2}, \ldots, m_{z}\right)$ is a partition of $n+1$ and hence a unipotent class in $G\left(A_{n}\right)_{q}$ and $\left(g_{1}, \ldots, g_{a}\right)=\left(m_{1}, \ldots, m_{z}\right)^{*}$ then we claim that $X=A_{g_{1}-1} \ldots A_{g_{a}-1} T_{m_{1}-1}$ (where $A_{0}=1$ by convention) represents the $\sim_{t}$ class of the connected centralizer of some semisimple element in $G\left(A_{n}\right)_{p}$ and that $f_{A}(X)=\mathcal{C}$. Now using Lemma 3.1 we can see that the proposed connected centralizer does have rank $n$. Also, since the number of parts in a partition is equal to the largest part of the dual of that partition, when we use Algorithm 2.2 we have at least as many deleted vertices $\left(m_{1}\right)$ as connected components $\left(m_{2}\right)$. Thus we can find a connected centralizer of the required type and it follows immediately from the lemma that $f_{A}$ will map it to $\mathcal{C}$. Therefore the map is indeed bijective.

Now recall that if $\mathcal{C}=\left(m_{1}, \ldots, m_{z}\right) \in \operatorname{uccl}_{q}(A)$ then $o_{e}(\mathcal{C})=\min \left\{q^{e}: q^{e} \geq m_{1}\right\}$. By the previous paragraph we see that the corresponding connected centralizer $X$ comes from a Dynkin diagram with $m_{1}$ deleted vertices. Thus $o_{\min }\left(X, A_{n}\right)=m_{1}$ and $q^{\left(X, A_{n}\right)}=\min \left\{q^{e}: q^{e} \geq m_{1}\right\}=o_{e}(\mathcal{C})$. With property $(\mathrm{C}+)$ proven we note that if $p \neq q$ then $q^{\left(X, A_{n}\right)}=q^{i}$ is coprime to $p$ and to construct $s \in G\left(A_{n}\right)_{p}$ we simply label the $m_{1}$ vertices so that at most one vertex has label greater than one and the sum of the labels is $q^{\left(X, A_{n}\right)}$. Thus we have $o(s)=q^{\left(X, A_{n}\right)}$ and it only remains to prove property (B). So take $\mathcal{C}=\left(m_{1}, \ldots, m_{z}\right) \in \operatorname{uccl}_{q}(A)$ and let $\left(g_{1}, \ldots, g_{a}\right)=\left(m_{1}, \ldots, m_{z}\right)^{*}$ 
and $X=f_{A}^{-1}(\mathcal{C})$, then $a=m_{1}$ and $X=A_{g_{1}-1} \ldots A_{g_{a}-1} T_{m_{1}-1}$. Therefore

$$
\begin{aligned}
\operatorname{dim} X & =\sum_{i=1}^{a} \operatorname{dim} A_{g_{i}-1}+\left(m_{1}-1\right)=\sum_{i=1}^{a}\left(g_{i}^{2}-1\right)+\left(m_{1}-1\right) \\
& =\sum_{i=1}^{a} g_{i}^{2}-a+\left(m_{1}-1\right)=\sum_{i=1}^{a} g_{i}^{2}-1=\text { c.dim } \mathcal{C} .
\end{aligned}
$$

\section{Type $C$}

In this section we will deal with groups of type $C$. We begin, as in type $A$, with the extended Dynkin diagram of type $\widetilde{C_{n}}$, together with the weights obtained from the highest root.

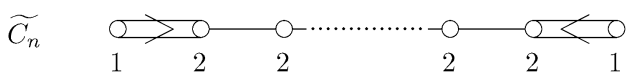

Now before proceeding we should make clear our conventions regarding groups of type $C$ with small rank. $C_{1}$ can be defined and is isomorphic to $A_{1}$, however as subgroups of a larger $C_{n}$ we know that $C_{1}$ and $A_{1}$ are not conjugate; thus these subgroups are viewed as different.

Now using Algorithm 2.2 and the extended Dynkin diagram allows us to list the $\sim_{t}$ classes of connected centralizers of semisimple elements. We find that all connected centralizers have the form $A_{n_{1}} \ldots A_{n_{r}} C_{m_{1}} C_{m_{2}} T_{l}$ where for convenience $n_{1} \geq \ldots \geq n_{r}$ and $m_{1} \geq m_{2} \geq 0$. Also note that the number of vertices removed is $l+1$ and this is always at least one more than the number of subgraphs of type $A$ left after the removals, which is $r$. Finally, observe that all primes are admissible with respect to any connected centralizer with $m_{2}=0$, but for connected centralizers with two factors of type $C$ only 2 is admissible. This follows since if $m_{2}=0$ then when obtaining the connected centralizer from Algorithm 2.2 at least one deleted vertex has weight 1; we can label that vertex such that the weighted sum of the labels is $q^{i}$ for some $i$. If all deleted vertices have weight 2 then the weighted sum must be even and so can only be a prime power if the prime is two.

Thus all the sets $\operatorname{Cent}_{q}(C)$, for odd $q$, are the same; they all equal

$$
\begin{gathered}
\left\{A_{n_{1}} \ldots A_{n_{r}} C_{m_{1}} T_{l}: n_{1}, \ldots, n_{r}, m_{1}, l \in \mathbb{N} \cup\{0\}\right. \\
\text { where } \left.n_{1} \geq \ldots \geq n_{r}, r \leq l, \text { and } m_{1} \geq 0\right\}
\end{gathered}
$$

The set $\mathrm{Cent}_{2}(C)$ equals

$$
\begin{gathered}
\left\{A_{n_{1}} \ldots A_{n_{r}} C_{m_{1}} C_{m_{2}} T_{l}: n_{1}, \ldots, n_{r}, m_{1}, m_{2}, l \in \mathbb{N} \cup\{0\},\right. \\
\text { where } \left.n_{1} \geq \ldots \geq n_{r}, r \leq l, \text { and } m_{1} \geq m_{2} \geq 0\right\}
\end{gathered}
$$

Note that if $q$ is odd then $\operatorname{Cent}_{q}(C)$ is the subset of $\operatorname{Cent}_{2}(C)$ where $m_{2}=0$. 
Next we consider unipotent conjugacy classes in type $C_{n}$ over characteristic $q$. Following [3] we see that these are parameterized by symbols $p_{1} m_{1} m_{1} \cdots p_{z} \chi_{z}$. Here $p_{1}{ }^{m_{1}} \ldots p_{z}{ }^{m_{z}}$ is a partition of $2 n$ given by the Jordan structure on the natural module, where each odd part has even multiplicity and $\chi: p_{j} \mapsto \chi_{j}$ is a function from the parts of $p_{1}{ }^{m_{1}} \cdots p_{z}{ }^{m_{z}}$ to the natural numbers. The function $\chi$ satisfies the following rule when the characteristic is 2 :

$$
\chi(p)= \begin{cases}\frac{1}{2}(p-1) & \text { if } p \text { is odd } \\ \frac{1}{2} p & \text { if } p \text { is even and has odd multiplicity } \\ \frac{1}{2}(p-2) \text { or } \frac{1}{2} p & \text { if } p \text { is even and has even multiplicity. }\end{cases}
$$

When $q$ is odd the rule is much simpler:

$$
\chi(p)= \begin{cases}\frac{1}{2}(p-1) & \text { if } p \text { is odd } \\ \frac{1}{2} p & \text { if } p \text { is even. }\end{cases}
$$

By identifying members $\mathbf{p} \in \operatorname{uccl}_{q}(C)$ with the appropriate symbol we again get a bijective representation and we will use this identification from now on. As with type $A$ this representation has certain advantages. For example the symbols can be seen diagrammatically as follows (we call these Hesselink diagrams since they are simply a way of visualizing the symbols used in [3]).

\section{Example}

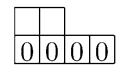

$2_{0}^{2} 1_{0}^{2} \in \operatorname{uccl}_{2}(C)$

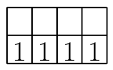

$2_{1}^{4} \in \operatorname{uccl}_{q}(C)$ for any $q$

The columns in the diagrams are in one-to-one correspondence with the parts in the partitions: the $i$ th column contains $p_{i}$ squares if and only if the $i$ th part has size $p_{i}$. The value in the bottom square of the $i$ th column is $\chi\left(p_{i}\right)$. This helps to visualize an operation we will need to perform later. Another advantage to the symbols is that by definition $r(\mathbf{p})=n=\frac{1}{2}|\mathbf{p}|$; we also know from [3] that if $\mathbf{p}=p_{1 \chi_{1}}^{m_{1}} \cdots p_{z \chi_{z}}^{m_{z}}$, then

$$
\mathbf{c} \operatorname{dim} \mathbf{p}=\sum_{i=1}^{z}\left(i p_{i}-\chi\left(p_{i}\right)\right) .
$$

As with type $A$ we see that $o_{e}(\mathbf{p})=\min \left\{q^{i} ; q^{i} \geq p_{1}\right\}$, but again we must be careful to avoid ambiguity by always stating $q$ when talking about $o_{e}(\mathbf{p})$.

Finally we note that partitions in which each odd part has even multiplicity are in one-to-one correspondence with pairs of partitions $(\alpha ; \beta)$ where $2|\alpha|+|\beta|=2 n$ and $\beta$ has distinct even parts. The original partition is obtained by taking the parts from $\alpha$ twice, together with the parts from $\beta$. Now with all the notation in place we obtain the following result. 
Theorem 5.1 For each prime $q$, there exists a function $f_{C, q}: \operatorname{Cent}_{q}(C) \rightarrow \operatorname{uccl}_{q}(C)$ satisfying properties $(A)$ and $(B)$. Furthermore, if $X \in \operatorname{Cent}_{q}(C)$, then $q^{\left(X, C_{n}\right)}=$ $o_{e}\left(f_{C, q}(X)\right)$ unless $(q, l)=\left(2,2^{i}\right)$ for some $i$ and one of the following holds:

1. $X$ has one $C$-factor and $l>r$;

2. $X$ has two $C$-factors and $l>r+1$.

In these cases we have $2^{\left(X, C_{n}\right)}=2 . o_{e}\left(f_{C, q}(X)\right)$. Finally, if $p \neq q$ then there exists $a$ semisimple element $s \in G\left(C_{n}\right)_{p}$ such that $C_{G\left(C_{n}\right)_{p}}(s) \sim_{t} X$ and $o(s)=q^{\left(X, C_{n}\right)}$.

Before proving this result we should say something about the importance of admissible primes. The reader may be wondering if the assumption that $q$ is admissible with respect to $X$ is really needed. Although we have no conceptual reason for it, we can prove that the result may fail if $X \notin \operatorname{Cent}_{q}(C)$. So let $q$ be an odd prime and note that $C_{2} C_{1} \notin \operatorname{Cent}_{q}(C)$. Below we list all the members of $\operatorname{uccl}_{q}(C)$. Recall that such classes are parameterized by pairs of partitions $(\alpha ; \beta)$ where $2|\alpha|+|\beta|=6$ and $\beta$ has distinct even parts. The Jordan structure is obtained by taking the parts from $\alpha$ twice, together with the parts from $\beta$.

\begin{tabular}{|l|l|c||l|l|c|}
\hline$(\alpha ; \beta)$ & Class & $\operatorname{dim} C_{G\left(C_{3}\right)_{q}}(u)$ & $(\alpha ; \beta)$ & Class & $\operatorname{dim} C_{G\left(C_{3}\right)_{q}}(u)$ \\
\hline$(3 ; 0)$ & $3_{1}^{2}$ & 7 & $(1,1 ; 2)$ & $2_{1} 1_{0}^{4}$ & 15 \\
$(2,1 ; 0)$ & $2_{1}^{2} 1_{0}^{2}$ & 11 & $(1 ; 4)$ & $4_{2} 1_{0}^{2}$ & 7 \\
$(1,1,1 ; 0)$ & $1_{0}^{6}$ & 21 & $(0 ; 2,4)$ & $4_{2} 2_{1}$ & 5 \\
$(2 ; 2)$ & $2_{1}^{3}$ & 9 & $(0 ; 6)$ & $6_{3}$ & 3 \\
\hline
\end{tabular}

This demonstrates that no class has centralizer dimension equal to $13=\operatorname{dim} C_{2} C_{1}$. So we cannot produce a function $g: \mho \rightarrow \operatorname{uccl}_{q}(C)$ satisfying property (B) if $\mho \mho$ contains $C_{2} C_{1}$.

Proof The proof will proceed in three stages. The first two deal with defining $f_{C, q}$ and showing it satisfies properties (A) and (B) and the last looks at the values of $q^{\left(X, C_{n}\right)}$ and the existence of $s$.

Stage 1: Defining $f_{C, q}(X)$ when $X=A_{n_{1}} \ldots A_{n_{r}} T_{l}$.

If $\mathbf{n}=\left(n_{1}, \ldots, n_{r}\right)$, where $n_{1} \geq \cdots \geq n_{r} \geq 0$, and if we set the $\chi$-values of $2\left(l, \mathbf{n}^{*}\right)$ to take their largest possible values, then we define

$$
\begin{aligned}
f_{C, q}\left(A_{n_{1}} \ldots A_{n_{r}} T_{l}\right) & =2\left(l, \mathbf{n}^{*}\right) \\
& =2 l_{l} 2 r_{r}^{n_{r}} 2(r-1)_{r-1}^{n_{r-1}-n_{r}} \cdots 2_{1}^{n_{1}-n_{2}} .
\end{aligned}
$$

That the two partitions are equal is easily seen. It is also easily seen that $\operatorname{rank} X=$ $\frac{1}{2}\left|f_{C, q}(X)\right|=r\left(f_{C, q}(X)\right)$. So we turn to the equality of centralizer dimensions. We begin by noting that $f_{C, q}\left(T_{l}\right)=2 l_{l}$ and

$$
\text { c. } \operatorname{dim} 2 l_{l}=2 l-l=\operatorname{dim} T_{l} .
$$


Next observe that replacing $A_{n_{i}}$ by $A_{n_{i}+1}$ in $X$ will increase $\operatorname{dim} X$ by $2 n_{i}+3$. Also observe that increasing the part and $\chi$-value of $f_{C, q}(X)$ in position $n_{i}+2$ by 2 and 1 respectively will increase c.dim $f_{C, q}(X)$ by $2\left(n_{i}+2\right)-1=2 n_{i}+3$. So starting from $X=T_{l}=A_{0}^{r} T_{l}$ and increasing the rank of each $A$-factor step-by-step we see that c.dim $f_{C, q}(X)=\operatorname{dim} X$ for all $X$ of the form $A_{n_{1}} \ldots A_{n_{r}} T_{l}$. Therefore the restriction of $f_{C, q}$ defined above satisfies properties (A) and (B). Note that the $X \in \operatorname{Cent}_{q}(C)$ for all $q$ and clearly the image is contained in $\operatorname{uccl}_{q}(C)$ for any $q$.

Stage 2: Defining $f_{C, q}(X)$ for arbitrary $X$.

Here we define $f_{C, q}\left(A_{n_{1}} \ldots A_{n_{r}} C_{m_{1}} C_{m_{2}} T_{l}\right)$, where $m_{1}$ and $m_{2}$ are arbitrary. Unlike the above we will not give an explicit formula for $f_{C, q}(X)$ here, instead we will give an algorithm for producing it. Now we know what $f_{C, q}(X)$ is when the $C$-factors both have rank 0 . So begin with $\mathcal{X}=A_{n_{1}} \ldots A_{n_{r}} C_{m^{(1)}} C_{m^{(2)}} T_{l}$ where $m^{(1)}=m^{(2)}=0$; also let $m_{v}$ be the rank of the $v$ th $C$-factor in $X$. We will increase the $m^{(v)}$ until we reach $\left(m_{1}, m_{2}\right)$ (it should be made clear here that we are treating the connected centralizers as entirely formal strings of characters; our manipulations of them are not intended to correspond to anything group theoretic or geometric, although we do not discount the possibility that such an interpretation may be discovered). We will also give the corresponding alterations to make to $f_{C, q}(\mathcal{X})$. So proceed as follows.

Step one If $m_{2}=m^{(2)}$ then go to Step two. Otherwise increase $m^{(1)}$ and $m^{(2)}$ by 1 , then replace the parts $2 s_{s} 2 t_{t}$ of $f_{C, q}(\mathcal{X})$ in positions $2 m^{(2)}-1$ and $2 m^{(2)}$ by $2(s+1)_{s}^{2}$ if $s=t$, by $(2 s+1)_{s}^{2}$ if $s=t+1$ and by $2 s_{s} 2(t+2)_{t+2}$ if $s \geq t+2$. Repeat. (Note that $s$ and $t$ are allowed to be zero here.)

Step two If $m_{1}=m^{(1)}$ then we are done. Otherwise increase $m^{(1)}$ by 1 , then replace the parts $2 s_{s} 2 t_{t}$ in positions $2 m^{(1)}-1$ and $2 m^{(1)}$ by $(2 s+1)_{s}^{2}$ if $s=t$, and by $2 s_{s} 2(t+$ $1)_{t+1}$ if $s \geq t+1$. Repeat. (Again $s$ and $t$ are allowed to be zero.)

Below we illustrate each of the possibilities for Step one and Step two diagrammatically. In each case the first column represents the part in position $2 m^{(2)}-1$ or $2 m^{(1)}-1$ as appropriate.
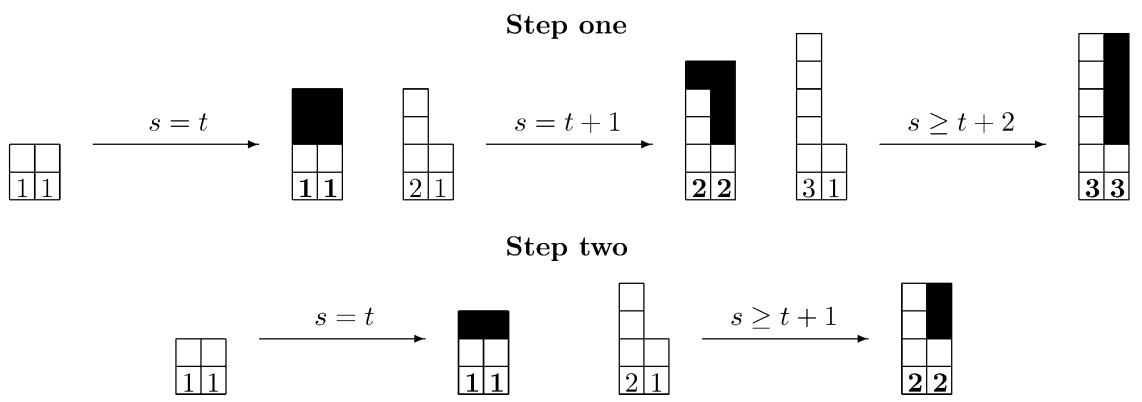

Now having given the algorithm, we must consider the question of whether the symbols produced by are valid members of $\operatorname{uccl}_{q}(C)$. First we observe that the descending order of the parts is preserved. This can be seen from the diagrams above for the parts in positions $2 m-1$ and $2 m\left(m=m^{(1)}\right.$ or $\left.m^{(2)}\right)$ and follows in general since the part in position $2 m$ is increased by at least 2 during Step one and at least 
1 during Step two and the part in position $2 m-1$ is increased by at most 2 during Step one and at most 1 during Step two. This is illustrated below. The values in bold are those which the algorithm has just acted upon, the black squares represent the increases to the parts. Note that the increase to the second part in each pair is never less than the increase to the first in the next pair.

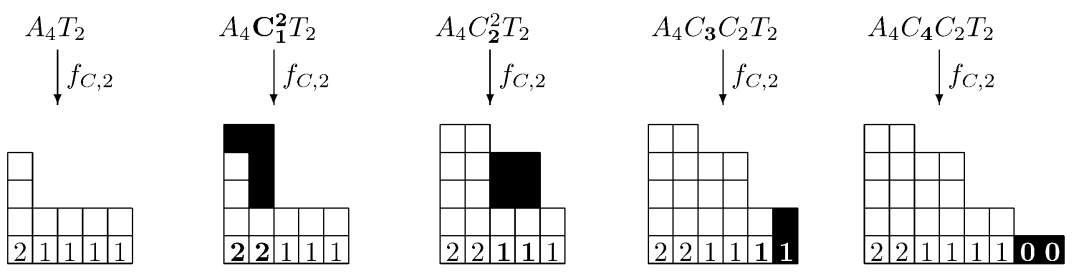

Next note that since we begin with valid symbols and any odd parts created come in pairs, we do get a partition with each odd part having even multiplicity. Also if $X \in \operatorname{Cent}_{q}(C)$ for odd $q$ then calculating $f_{C, q}$ will not require Step one and from this we see that the $\chi$-values place the images of the function in the appropriate $\operatorname{uccl}_{q}(C)$.

The final thing to check is that we do not produce parts of the same size, but with different $\chi$-values. From the steps of the algorithm we see that this cannot happen with the parts in positions $2 m-1$ and $2 m$. We must rule the situation out for parts in positions $2 m$ and $2(m+1)-1=2 m+1$. It could only happen if the parts were even, since the size determines the $\chi$-value for odd parts. From the algorithm we see that the only way to produce even parts of the same size in positions $2 m$ and $2(m+1)-1=2 m+1$ is to begin with even parts of equal size and use Step one consecutively. However in that case we produce the same $\chi$-values for equal-sized parts. Thus the algorithm does produce valid members of $\operatorname{uccl}_{q}(C)$ and our $f_{C, q}$ is well-defined.

We are now ready to prove the equality of ranks and centralizer dimensions. We recall that rank $\mathcal{X}=\frac{1}{2}\left|f_{C, q}(\mathcal{X})\right|=r\left(f_{C, q}(\mathcal{X})\right)$ held before the algorithm and for each increase in the rank of $\mathcal{X}$, the algorithm increases the size of the partition $f_{C, q}(\mathcal{X})$ by twice that amount. Step one increases rank $\mathcal{X}$ by 2 each time and $\left|f_{C, q}(\mathcal{X})\right|$ by 4 ; Step two increases $\operatorname{rank} \mathcal{X}$ by 1 and $\left|f_{C, q}(\mathcal{X})\right|$ by 2 . Thus the equality is preserved by the algorithm. To prove that the equality c.dim $f_{C, q}(\mathcal{X})=\operatorname{dim} \mathcal{X}$ is also preserved we first note that

$$
\operatorname{dim} C_{m}^{t}-\operatorname{dim} C_{m-1}^{t}=t(4 m-1), \quad t \in\{1,2\}
$$

Now we must show that the changes made to the symbol by our algorithm match this increase. So, in Step one we begin with $2 s_{s} 2 t_{t}$ in positions $2 m-1$ and $2 m$. If $s=t$ we increase both these parts by 2 , but leave the $\chi$-values unchanged. This gives a centralizer dimension increase of

$$
2(2 m-1)+2(2 m)=2(4 m-1),
$$

as required. If $s=t+1$ then we increase the part in position $2 m-1$ by 1 , but leave the $\chi$-value unchanged. We also increase the part and $\chi$-value in position $2 m$ by 3 
and 1 respectively. These increases give a centralizer dimension increase of

$$
1(2 m-1)+3(2 m)-1=2(4 m-1),
$$

again as required. Lastly, if $s \geq t+2$ then we increase the part and $\chi$-value in position $2 m$ by 4 and 2 respectively. This gives a centralizer dimension increase of

$$
4(2 m)-2=2(4 m-1)
$$

as required.

Finally, in Step two we begin with $2 s_{s} 2 t_{t}$ in positions $2 m-1$ and $2 m$ then increase both parts by 1 and leave the $\chi$-values unchanged if $s=t$ and increase the part and $\chi$-value in position $2 m$ by 2 and 1 respectively if $s \geq t+1$. In both cases we get a centralizer dimension increase of $4 m-1$, as required. Thus the equality of centralizer dimensions is preserved by the algorithm and holds for all $X$. So we have now constructed our function and shown that it satisfies properties (A) and $(\mathrm{B})$.

Stage 3: The values of $q^{\left(X, C_{n}\right)}$.

First recall that if $J$ is the size of the largest Jordan block of $f_{C, q}(X)$, then $o_{e}\left(f_{C, q}(X)\right)=\min \left\{q^{e}: q^{e} \geq J\right\}$. Now proceed with the case analysis

Case 1: $X$ has no $C$-factor (so $X \in \operatorname{Cent}_{q}(C)$ for all $q$ ).

Here we see that $o_{e}\left(f_{C, q}(X)\right)=\min \left\{q^{e}: q^{e} \geq 2 l\right\}$. Furthermore we see that the two outer vertices of weight 1 must have been deleted from the extended Dynkin diagram $\widetilde{C_{n}}$ when getting $X$ from Algorithm 2.2. Since $l-1$ vertices with weight 2 were also deleted we see that $o_{\min }\left(X, C_{n}\right)=2 l$. This clearly gives us $q^{\left(X, C_{n}\right)}=\min \left\{q^{e}: q^{e} \geq 2 l\right\}=o_{e}\left(f_{C, q}(X)\right)$. Now, if $p \neq q$ then $q^{\left(X, C_{n}\right)}=q^{i}$ is coprime to $p$ and we can proof the existence of an $s \in G\left(C_{n}\right)_{p}$ by labeling the vertices so that at most one vertex has label greater than one and the sum of the labels is $q^{\left(X, C_{n}\right)}$.

Case 2: $X$ has one $C$-factor (so $X \in \operatorname{Cent}_{q}(C)$ for all $q$ ).

From the formula given in Stage 1 of this proof and Step two of the algorithm in Stage 2 we see that $J=2 l+1$ if $l=r$, and $2 l$ otherwise. To obtain $X$ from Algorithm 2.2 we have deleted $l$ vertices of weight 2 and one of weight 1 in $\widetilde{C_{n}}$. So $o_{\min }\left(X, C_{n}\right)=2 l+1$ and if $l=r$ we clearly have $q^{\left(X, C_{n}\right)}=\min \left\{q^{e}: q^{e} \geq\right.$ $2 l+1\}=o_{e}\left(f_{C, q}(X)\right)$. If we have $l>r$ then $q^{\left(X, C_{n}\right)}=\min \left\{q^{e}: q^{e} \geq 2 l+1\right\}=$ $\min \left\{q^{e}: q^{e} \geq 2 l\right\}=o_{e}\left(f_{C, q}(X)\right)$ unless $(q, l)=\left(2,2^{i}\right)$ for some $i$, when we get $2^{\left(X, C_{n}\right)}=2 . o_{e}\left(f_{C, q}(X)\right)$. Lastly, if $p \neq q$ then we proceed as above to obtain a semisimple element of order $q^{\left(X, C_{n}\right)}$.

Case 3: $X$ has two $C$-factors (so $X$ is only contained in $\mathrm{Cent}_{2}(C)$ ).

As in Case 2 we see that $J=2 l+2$ if $l=r, 2 l+1$ if $l=r+1$, and $2 l$ otherwise. We also see that to obtain $X$ from Algorithm 2.2 we have deleted $l+1$ vertices of weight 2 in $\widetilde{C_{n}}$, so $o_{\min }\left(X, C_{n}\right)=2 l+2$. Since the only admissible prime here is 2 we now set $q=2$.

There are three subcases to deal with, so first let $l=r$ and then $2^{\left(X, C_{n}\right)}=\min \left\{2^{e}\right.$ : $\left.2^{e} \geq 2 l+2\right\}=o_{e}\left(f_{C, q}(X)\right)$. Next take $l=r+1$ and observe that since $2 l+1$ is odd we have $2^{\left(X, C_{n}\right)}=\min \left\{2^{e}: 2^{e} \geq 2 l+2\right\}=\min \left\{2^{e}: 2^{e} \geq 2 l+1\right\}=o_{e}\left(f_{C, q}(X)\right)$. Finally if $l>r+1$ then since $2 l+1$ is odd we have $2^{\left(X, C_{n}\right)}=\min \left\{2^{e}: 2^{e} \geq\right.$ 
$2 l+2\}=\min \left\{2^{e}: 2^{e} \geq 2 l\right\}=o_{e}\left(f_{C, q}(X)\right)$ unless $l=2^{i}$ for some $i$, when we get $2^{\left(X, C_{n}\right)}=2 . o_{e}\left(f_{C, q}(X)\right)$. Lastly, if $p \neq 2$ then we again proceed as above to obtain a semisimple element of order $2^{\left(X, C_{n}\right)}$.

We conclude this section by noting that the above proof provides a blueprint that we will follow in several more situations. As a result we will sometimes not give the details as fully as we have here. This should not be a problem as the proofs are mostly straightforward, the difficulty is in knowing how to define our functions in the first place.

\section{Type $D$}

In this section we will deal with groups of type $D$. We begin as before with the extended Dynkin diagram of type $\widetilde{D_{n}}$, together with the weights obtained from the highest root.

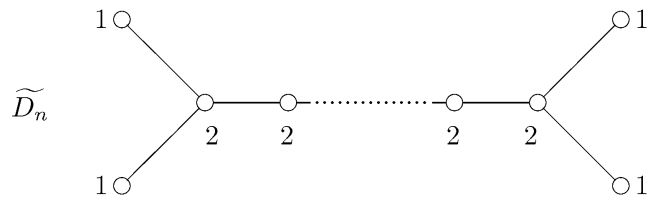

Once again we should make clear our conventions regarding groups with small rank. $D_{3}, D_{2}$ and $D_{1}$ can be defined and are isomorphic to $A_{3}, A_{1}^{2}$ and $T_{1}$ respectively, however as subgroups of a larger $D_{n}$ we know that $D_{3}$ and $A_{3}$ are not conjugate and nor are $D_{2}$ and $A_{1}^{2}$; thus these subgroups are viewed as different. $D_{1}$ and $T_{1}$ are viewed as the same. We take $D_{0}$ to be the trivial group.

Using Algorithm 2.2 allows us to list the $\sim_{t}$ classes of connected centralizers of semisimple elements. However before giving the possibilities we must first observe that since $T_{l}=k^{l}=T_{1}^{l}$ (here $k$ is the field our group is defined over) and $T_{1}=D_{1}$ the number of factors of type $D$ is not an invariant: $T_{l}$ may be written as $D_{1}^{a} T_{l-a}$ for any $0 \leq a \leq l$ (of course this was also true for types $A$ and $C$, but since in those cases there were no other $D$-factors we simply choose to always write $T_{l}$ ). With this in mind we note that the connected centralizers of semisimple elements in type $D$ all have the form $X=A_{n_{1}} \ldots A_{n_{r}} D_{m_{1}} \ldots D_{m_{d}}$, where $n_{1} \geq \ldots \geq n_{r}$ and $m_{1} \geq \ldots \geq m_{d}$ (it is convenient later to write $T_{l}$ as $D_{1}^{l}$ ). Algorithm 2.2 also shows that the number of $D$-factors with rank at least 2 (denoted $\delta$ ) is less than or equal to 2 and that the number of $A$-factors $(r)$ can be no more than the number of deleted vertices minus one, which equals the dimension of the central torus, i.e. $d-\delta$.

Now observe that all primes are admissible with respect to any connected centralizer with $\delta \leq 1$, but for connected centralizers with $\delta=2$ only 2 is admissible. This follows since if $\delta \leq 1$ then when obtaining the connected centralizer from Algorithm 2.2 at least one deleted vertex has weight 1; we can label that vertex such that the weighted sum of the labels is $q^{i}$ for some $i$. If all deleted vertices have weight 2 then the weighted sum must be even and so can only be a prime power if the prime is two. 
Thus all the sets $\operatorname{Cent}_{q}(D)$, for odd $q$, are the same; they all equal

$$
\begin{aligned}
& \left\{A_{n_{1}} \ldots A_{n_{r}} D_{m_{1}} D_{1}^{l}: n_{1}, \ldots, n_{r}, m_{1}, l \in \mathbb{N} \cup\{0\},\right. \\
& \text { where } \left.n_{1} \geq \ldots \geq n_{r}, r \leq l, \text { and } m_{1} \geq 0\right\} .
\end{aligned}
$$

The set $\operatorname{Cent}_{2}(D)$ equals

$$
\begin{aligned}
& \left\{A_{n_{1}} \ldots A_{n_{r}} D_{m_{1}} D_{m_{2}} D_{1}^{l}: n_{1}, \ldots, n_{r}, m_{1}, m_{2}, l \in \mathbb{N} \cup\{0\},\right. \\
& \text { where } \left.n_{1} \geq \ldots \geq n_{r}, r \leq l, \text { and } m_{1} \geq m_{2} \geq 0\right\} .
\end{aligned}
$$

Next we consider unipotent conjugacy classes in type $D_{n}$ over odd characteristic $q$ (postponing the treatment of characteristic 2 until later). As before, from [3] we see that these are parameterized by symbols $p_{1 \chi_{1}}^{m_{1}} \cdots p_{z \chi_{z}}^{m_{z}}$, where $p_{1}{ }^{m_{1}} \cdots p_{z}{ }^{m_{z}}$ is a partition of $2 n$ given by the Jordan structure on the natural module. This time each even part has even multiplicity and $\chi: p_{j} \mapsto \chi_{j}$ is a function from the parts of $p_{1}{ }^{m_{1}} \ldots p_{z}{ }^{m_{z}}$ to the natural numbers satisfying

$$
\chi(p)= \begin{cases}\frac{1}{2}(p+1) & \text { if } p \text { is odd } \\ \frac{1}{2} p & \text { if } p \text { is even }\end{cases}
$$

By identifying members $\mathbf{p} \in \operatorname{uccl}_{q}(D)$ with the appropriate symbol we again get a bijective representation and we will use this identification from now on. We can define Hesselink diagrams in exactly the same way as we did in type $C$ and they will again help to visualize the operations of our functions. Also by definition $r(\mathbf{p})=n=\frac{1}{2}|\mathbf{p}|$, from [3] we see that if $\mathbf{p}=p_{1} \chi_{1}^{m_{1}} \cdots p_{z \chi_{z}}^{m_{z}}$, then

$$
\text { c.dim } \mathbf{p}=\sum_{i=1}^{z}\left(i p_{i}-\chi\left(p_{i}\right)\right)
$$

and $o_{e}(\mathbf{p})=\min \left\{q^{i} ; q^{i} \geq p_{1}\right\}$. We repeat the now standard warning to avoid ambiguity by always stating $q$ when talking about $o_{e}(\mathbf{p})$.

Finally we note that if all parts of the partition are even then there are two classes associated to that partition; we will treat these classes as the same. Now with all the notation in place we obtain the following result.

Theorem 6.1 For each odd prime $q$, there exists a function $f_{D, q}: \operatorname{Cent}_{q}(D) \rightarrow$ $\operatorname{uccl}_{q}(D)$ satisfying properties $(A),(B)$ and $(C+)$. Furthermore, if $p \neq q$ then there exists a semisimple element $s \in G\left(D_{n}\right)_{p}$ such that $C_{G\left(D_{n}\right)_{p}}(s) \sim_{t} X$ and $o(s)=q^{\left(X, D_{n}\right)}$.

Proof The proof will proceed along the lines established in the proof of Theorem 5.1. Stage 1: Defining $f_{D, q}\left(A_{n_{1}} \ldots A_{n_{r}} D_{m_{1}} D_{1}^{l}\right)$ when $r \leq l$ and $2 m_{1}-1 \geq n_{1} \geq \cdots \geq$ $n_{r} \geq 0$.

First note that $2 m_{1}-1 \geq 0$ and $m_{1} \in \mathbb{Z}$ imply that $m_{1} \geq 1$, so $d$ (the number of $D$-factors) is $l+1$. Now set $\mathbf{n}=\left(n_{1}, \ldots, n_{r}\right)$, then if $2 m_{1}-1 \geq n_{1} \geq \cdots \geq n_{r} \geq 0$ 
and $r \leq l$ we define

$$
\begin{aligned}
f_{D, q}\left(A_{n_{1}} \ldots A_{n_{r}} D_{m_{1}} D_{1}^{l}\right)= & 2\left(l+1,\left(2 m_{1}-1, \mathbf{n}\right)^{*}\right)-1 \\
= & (2 l+1)_{l+1}(2 r+1)_{r+1}^{n_{r}}(2 r-1)_{r}^{n_{r-1}-n_{r}} \ldots \\
& \times 3_{2}^{n_{1}-n_{2}} 1_{1}^{2 m_{1}-1-n_{1}} .
\end{aligned}
$$

That the two partitions are equal is easily seen. It is also easily seen that $\operatorname{rank} X=$ $\frac{1}{2}\left|f_{D, q}(X)\right|=r\left(f_{D, q}(X)\right)$. So we turn to the equality of centralizer dimensions. We begin by noting that $f_{D, q}\left(D_{1}^{l+1}\right)=(2 l+1)_{l+1} 1_{1}$. We then see that

$$
\text { c. } \operatorname{dim}(2 l+1)_{l+1} 1_{1}=(1(2 l+1)-(l+1))+(2(1)-1)=l+1=\operatorname{dim} D_{1}^{l+1} .
$$

Next observe that increasing $m_{1}$ by 1 will increase $\operatorname{dim} X$ by $4 m_{1}+1$. To match this we observe that increasing both the parts and the $\chi$-values in positions $2 m_{1}+1$ and $2 m_{1}+2$ by 1 increases c.dim $f_{D, q}(X)$ by $\left(1\left(2 m_{1}+1\right)-1\right)+\left(1\left(2 m_{1}+2\right)-1\right)=$ $4 m_{1}+1$. So we get c.dim $f_{D, q}(X)=\operatorname{dim} X$ for all $X$ of the form $D_{m_{1}} D_{1}^{l}$. Finally we note that increasing $n_{i}$ by 1 will increase $\operatorname{dim} X$ by $2 n_{i}+3$, and increasing the part and $\chi$-value in position $n_{i}+2$ by 2 and 1 respectively will increase c.dim $f_{D, q}(X)$ by $2\left(n_{i}+2\right)-1=2 n_{i}+3$. Therefore the restriction of $f_{D, q}$ defined above sat-

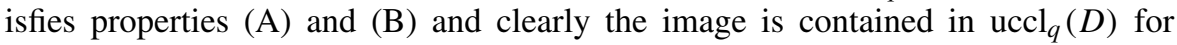
any odd $q$.

Stage 2: Defining $f_{D, q}(X)$ for arbitrary $X$.

Take $X \in \operatorname{Cent}_{q}(D)$ with $q$ odd: if it satisfies the conditions of the previous stage then the image has already been defined, if not we will construct an element $\widehat{X}$ that does. Recall that $0 \leq \delta \leq 1$ since $q$ is odd (here $\delta$ is the number of $D$ factors with rank greater then 1) and since we always have $r \leq d-\delta$ we see that $r \leq l(=d-1)$ can only be broken if $\delta=0$ and $r=d$. Thus if $X$ fails to satisfy our first condition, adding a factor of type $D_{1}$ will give an element $\widetilde{X}$ which does (as in the previous section all operations of this kind are considered formal manipulations of strings of characters). If $X$ does satisfy $r \leq d-1$ then we set $\widetilde{X}=X$. Now if $\widetilde{X}$ fails to satisfy $2 \widetilde{m_{1}}-1 \geq \widetilde{n_{1}}$ we can simply increase $\widetilde{m_{1}}$ and construct an element $\widehat{X}$ which does. Note that increasing $\widetilde{m_{1}}$ in this way may increase $\delta$ from 0 to 1 , thus strengthening the condition $r \leq d-\delta$. However this condition will still hold since $\delta \leq 1$ gives $d-1 \leq d-\delta$ and therefore $r \leq d-1$ (which we started with and which clearly remains satisfied when $\widetilde{m_{1}} \geq 1$ is increased) implies $r \leq d-\delta$.

We now observe that $f_{D, q}(\widehat{X})$ is defined by the previous stage and that $X$ can be recovered from $\widehat{X}$ simply by decreasing $\widehat{m_{1}}$ (possibly to zero to reverse the process of creating $\widetilde{X}$; recall that $D_{0}$ is the trivial group by convention). We will decrease $\widehat{m}_{1}$ in steps of size one and give the corresponding alterations to make to $f_{D, q}(\widehat{X})$ at each stage. (This is why we write $D_{1}^{l}$ instead of $T_{l}$ : if $X=A_{3} A_{2} D_{1}^{2}$ then $\widehat{X}=A_{3} A_{2} D_{2} D_{1}^{2}$ and the sequence of elements we get by decreasing $\widehat{m_{1}}$ is $A_{3} A_{2} D_{2} D_{1}^{2}, A_{3} A_{2} D_{1}^{3}$, $A_{3} A_{2} D_{1}^{2}$, but if we use $T_{l}$ s this sequence would be written $A_{3} A_{2} D_{2} T_{2}, A_{3} A_{2} T_{3}$, $A_{3} A_{2} T_{2}$. The sequence clearly looks more natural and is easier to specify with the first notation.) We proceed as follows. 


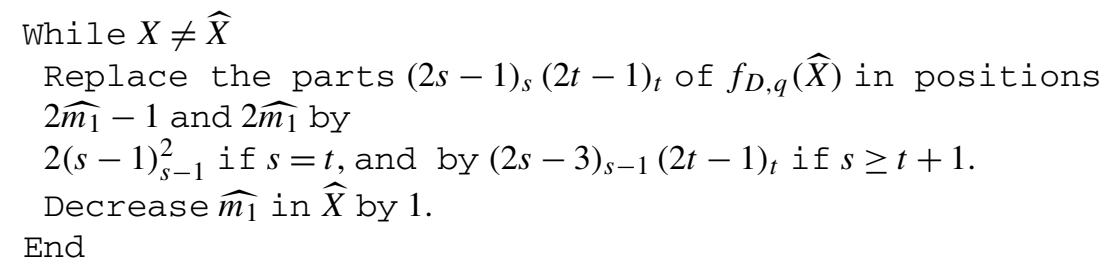

Below we illustrate our algorithm diagrammatically. In both cases the first column represents the part in position $2 \widehat{m_{1}}-1$.

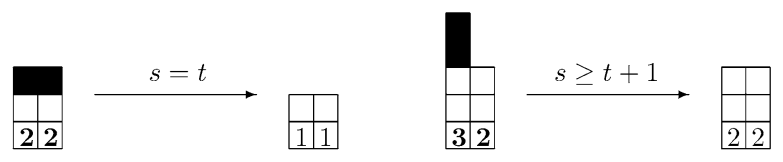

We conclude this stage by performing the appropriate checks. First observe that when constructing $\widehat{X}$ we did not require that $\widehat{m_{1}}$ was minimal with respect to $2 \widehat{m_{1}}-1 \geq$ $\widehat{n_{1}}$. So if $X$ satisfies $r \leq d-1$ and $2 m_{1}-1 \geq n_{1}$ and we form $X^{\prime}$ by increasing $m_{1}$, then both $f_{D, q}(X)$ and $f_{D, q}\left(X^{\prime}\right)$ will be determined by the formula given in the previous stage, however we could also take $X^{\prime}$ as $\widehat{X}$ and apply the algorithm to $f_{D, q}\left(X^{\prime}\right)=f_{D, q}(\widehat{X})$ to recover $f_{D, q}(X)$. Therefore we must show that the algorithm is consistent with the formula. So, if $f_{D, q}(X)$ has $2 m_{1}$ parts and $f_{D, q}\left(X^{\prime}\right)$ has $2 m_{1}^{\prime}$ parts, then the formula tells us that the parts in positions $2 m_{1}+1$ to $2 m_{1}^{\prime}$ of $f_{D, q}\left(X^{\prime}\right)$ all have size 1 . Thus to recover $X$ from $X^{\prime}$ we must use the algorithm $m_{1}^{\prime}-m_{1}$ times and this will decrease the final $2\left(m_{1}^{\prime}-m_{1}\right)$ parts of $f_{D, q}\left(X^{\prime}\right)$ to zero. Therefore the $f_{D, q}(X)$ determined by the algorithm is the same as the $f_{D, q}(X)$ determined by the formula and the function is well-defined.

Next we want to know that the symbols our procedure has produced are genuine elements of $\operatorname{uccl}_{q}(D)$. For this we must be sure that the descending order of the parts is maintained and that the even parts in the underlying partitions of our symbols still have even multiplicity. The same reasoning as in Theorem 5.1 shows that the descending order of the parts is maintained. The even parts have even multiplicity since the symbols of the form $f_{D, q}(\widehat{X})$ have only odd parts and any even parts created by the algorithm come in pairs.

Thirdly, we observe that at each stage of the algorithm the rank of the connected centralizer drops by 1 and the size of the underlying partition of the symbol drops by 2. Thus since the formula given in the previous stage satisfied $\operatorname{rank} \widehat{X}=$ $\frac{1}{2}\left|f_{D, q}(\widehat{X})\right|=r\left(f_{D, q}(\widehat{X})\right)$, we see that this holds in all cases.

Finally we come to the equality of centralizer dimensions. For this we notice that

$$
\operatorname{dim} D_{m-1}-\operatorname{dim} D_{m}=-(4 m-3) .
$$

Now we must show that the changes made to the symbol by our algorithm match this decrease; we have already seen that equality is satisfied before the algorithm is applied. We begin with $(2 s-1)_{s}(2 t-1)_{t}$ in positions $2 m-1$ and $2 m$. If $s=t$ we decrease both parts and $\chi$-values by 1 , otherwise we decrease the first part and $\chi$-value by 2 and 1 respectively. These give centralizer dimension decreases of $-((1(2 m-1)-1)+(1(2 m)-1))=-(4 m-3)$ and $-(2(2 m-1)-1)=-(4 m-3)$ 
respectively, as required. So since the equality c.dim $f_{D, q}(\widehat{X})=\operatorname{dim} \widehat{X}$ held before the algorithm, we see that it holds for all $X$. Thus our fully defined function satisfies properties (A) and (B).

Stage 3: The values of $q^{\left(X, D_{n}\right)}$.

Let $J$ be the size of the largest Jordan block of $f_{D, q}(X)$, then as in Theorem 5.1 we have $o_{e}\left(f_{D, q}(X)\right)=\min \left\{q^{e}: q^{e} \geq J\right\}$. To match this we must calculate $q^{\left(X, D_{n}\right)}$. Therefore we must find the value of $o_{\min }\left(X, D_{n}\right)$ under all possible conditions. Recall from the beginning of the section that $r \leq d-\delta, 0 \leq \delta \leq 1$ and the number of vertices deleted in the extended Dynkin diagram to get $X$ from Algorithm 2.2 is $d-\delta+1$.

First suppose that $\delta=0$ (so there is no $D$-factor of rank greater than one): here we delete $d+1$ vertices from the extended Dynkin diagram. Consider the following three subcases. If $d=r$ then the number of vertices deleted after applying Algorithm 2.2 is one more than the number of subgraphs of type $A$ left. We deduce that only two vertices of weight 1 have been deleted and $o_{\min }\left(X, D_{n}\right)=2(d-1)+1+1=2 d$. For example, the labels $0^{10010010}{ }_{0}^{1}$ give a semisimple element of order 6 with a connected centralizer of type $A_{3} A_{2}^{2} D_{1}^{3}$. If $d=r+1$ then the number of deleted vertices is two more than the number of subgraphs of type $A$ left. We deduce that three vertices of weight 1 have been deleted (we will use as many weight 1 vertices as we can since we are looking for the semisimple element with the smallest possible order) and $o_{\min }\left(X, D_{n}\right)=2(d-2)+1+1+1=2 d-1$, as when the labels ${ }_{1}^{1}{ }^{0010010}{ }_{0}^{1}$ give a semisimple element of order 7 with a connected centralizer of type $A_{2}^{3} D_{1}^{4}$. Finally if $d \geq r+2$ then all four vertices of weight 1 must have been deleted and we get $o_{\min }\left(X, D_{n}\right)=2(d-3)+1+1+1+1=2 d-2$.

Now let $\delta=1$ (so there is one $D$-factor with rank greater than one): here we delete $d$ vertices from the extended Dynkin diagram and have only two subcases to consider. If $d=r+1$ then the number of vertices deleted after applying Algorithm 2.2 is one more than the number of subgraphs of type $A$ left. Hence only one vertex of weight 1 has been deleted and $o_{\min }\left(X, D_{n}\right)=2(d-1)+1=2 d-1$. An example of this is given by the labels $0_{0}^{0010010}$ which give a semisimple element of order 5 with a connected centralizer of type $A_{2}^{2} D_{4} D_{1}^{2}$. If $d \geq r+2$ then at least two more vertices have been deleted than there are subgraphs of type $A$ left. We deduce that two vertices of weight 1 have been deleted and $o_{\min }\left(X, D_{n}\right)=2(d-2)+1+1=2 d-2$, as when the labels $0^{0010010}{ }_{1}^{1}$ give a semisimple element of order 6 with a connected centralizer of type $A_{2} A_{1} D_{4} D_{1}^{3}$. Overall we have shown the following:

$$
o_{\min }\left(X, D_{n}\right)= \begin{cases}2 d & \text { if } d=r \\ 2 d-1 & \text { if } d=r+1 \\ 2 d-2 & \text { if } d \geq r+2\end{cases}
$$

Now, if $p \neq q$ then $q^{\left(X, D_{n}\right)}=q^{i}$ is coprime to $p$ and we can construct $s \in G\left(D_{n}\right)_{p}$ by labeling the vertices so that at most one has label greater than one and the sum of the labels is $q^{\left(X, D_{n}\right)}$. This can always be done since we see by the above that at least one vertex of weight 1 will be deleted. 
Now recall from the previous stage that if $d \geq r+1$ then $X=\tilde{X}$. So having formed $\widehat{X}$, to regain $X$ we use the algorithm given in that stage and we note that we shall not decrease $\widehat{m_{1}}$ to 0 , since this would only be done to reverse adding a $D_{1}$. This process never took place when creating $\widehat{X}$ since we started with $X=\widetilde{X}$. Since $\widehat{m_{1}}$ is never decreased to 0 by the algorithm we also never decrease the parts in positions 1 and 2 . Therefore the largest part of $f_{D, q}(X)$ is equal to the largest part of $f_{D, q}(\widehat{X})$ which, by Stage 1 , is $2 l+1=2(d-1)+1=2 d-1$; hence $o_{e}\left(f_{D, q}(X)\right)=\min \left\{q^{e}: q^{e} \geq\right.$ $2 d-1\}$. At the same time we have $q^{\left(X, D_{n}\right)}=\min \left\{q^{e}: q^{e} \geq o_{\min }\left(X, D_{n}\right)\right\}$, where $o_{\min }\left(X, D_{n}\right)=2 d-1$ or $2 d-2$. However since $q$ is odd and $2 d-2$ is even we must have $q^{\left(X, D_{n}\right)}=\min \left\{q^{e}: q^{e} \geq 2 d-1\right\}=o_{e}\left(f_{D, q}(X)\right)$.

Thus the only case left to check is when $d=r$. This time when we form $\tilde{X}$ we get $\tilde{d}=r+1=d+1$. Now when we form $\widehat{X}$ we don't change the number of $D$ factors so $\widehat{d}=\tilde{d}=d+1$. Therefore from Stage 1 we see that the first two parts of $f_{D, q}(\widehat{X})$ are $(2 \widehat{l}+1)_{\widehat{l}+1}(2 r+1)_{r+1}=(2 \widehat{d}-1)_{\widehat{d}}(2 r+1)_{r+1}=(2 d+1)_{d+1}^{2}$. Now to recover $X$ from $\widehat{X}$ we will decrease $\widehat{m_{1}}$ to 0 and to match this we will, in particular, replace the parts $(2 d+1)_{d+1}^{2}$ in positions 1 and 2 by $2 d_{d}^{2}$. Therefore $J=2 d$, which equals $o_{\min }\left(X, D_{n}\right)$ by above, and we get $q^{\left(X, D_{n}\right)}=o_{e}\left(f_{D, q}(X)\right)$. So property $(\mathrm{C}+)$ has been demonstrated and the proof is complete.

We can now consider unipotent conjugacy classes in type $D_{n}$ over characteristic 2. Following [3], these are again parameterized by symbols $\mathbf{p}=p_{1}{ }_{1}^{t_{1}} \cdots p_{z}{ }_{z z}$ (this is a bijective representation and we will use this identification from now on). Here $p_{1}{ }^{t_{1}} \cdots p_{z}{ }^{t_{z}}$ is a partition of $2 n$ given by the Jordan structure, with an even number of parts and where each odd part has even multiplicity. The map $\chi: p_{j} \mapsto \chi_{j}$ from the parts of $p_{1}{ }^{t_{1}} \cdots p_{z}{ }^{t_{z}}$ to the natural numbers satisfies the following rule:

$$
\chi(p)= \begin{cases}\frac{1}{2}(p+1) & \text { if } p \text { is odd } \\ \frac{1}{2}(p+2) & \text { if } p \text { is even and has odd multiplicity } \\ \frac{1}{2} p \text { or } \frac{1}{2}(p+2) & \text { if } p \text { is even and has even multiplicity. }\end{cases}
$$

Here we should note that there are two classes associated to symbols where $\chi(p)=$ $\frac{1}{2} p$ for all parts $p$; we again treat these classes as the same.

Once again we get $r(\mathbf{p})=n=\frac{1}{2}|\mathbf{p}|$, if $\mathbf{p}=p_{1} \chi_{1}^{m_{1}} \cdots p_{z} \chi_{z}$, then

$$
\operatorname{codim} \mathbf{p}=\sum_{i=1}^{z}\left(i p_{i}-\chi\left(p_{i}\right)\right)
$$

and $o_{e}(\mathbf{p})=\min \left\{q^{i} ; q^{i} \geq p_{1}\right\}$. Now with all the notation confirmed we obtain the following result.

Theorem 6.2 There exists a function $f_{D, 2}: \operatorname{Cent}_{2}(D) \rightarrow \operatorname{uccl}_{2}(D)$ satisfying properties $(A),(B)$ and $(C+)$. Furthermore, if $p \neq 2$ then there exists a semisimple element $s \in G\left(D_{n}\right)_{p}$ such that $C_{G\left(D_{n}\right)_{p}}(s) \sim_{t} X$ and $o(s)=2^{\left(X, D_{n}\right)}$. 
Proof The structure of the proof should be familiar by now.

Stage 1: Defining $f_{D, 2}\left(A_{n_{1}} \ldots A_{n_{r}} D_{m_{1}}^{2} D_{1}^{l}\right)$ when $r \leq l$ and $2 m_{1}-1 \geq n_{1} \geq \cdots \geq$ $n_{r} \geq 0$.

First note that $2 m_{1}-1 \geq 0$ and $m_{1}=m_{2} \in \mathbb{Z}$ imply that $m_{2}=m_{1} \geq 1$ and so $d$ (the number of $D$-factors) is $l+2$. Now set $\mathbf{n}=\left(n_{1}, \ldots, n_{r}\right)$, then if $2 m_{1}-1 \geq n_{1} \geq$ $\cdots \geq n_{r} \geq 0$ and $r \leq l$ we have

$$
\begin{aligned}
f_{D, 2}\left(A_{n_{1}} \ldots A_{n_{r}} D_{m_{1}}^{2} D_{1}^{l}\right) & =2\left(l+1,\left(2 m_{1}-1, \mathbf{n}\right)^{*}\right) \\
& =2(l+1)_{l+2} 2(r+1)_{r+2}^{n_{r}} 2 r_{r+1}^{n_{r-1}-n_{r}} \ldots 4_{3}^{n_{1}-n_{2}} 2_{2}^{2 m_{1}-1-n_{1}} .
\end{aligned}
$$

It is easily seen that the two partitions are equal and properties (A) and (B) hold for this restriction by the same reasoning used for Theorems 5.1 and 6.1.

Stage 2: Defining $f_{D, 2}(X)$ for arbitrary $X$.

Take $X \in \operatorname{Cent}_{2}(D)$ : if it satisfies the conditions of the previous stage then the image has already been defined, if not we will again construct an element $\widehat{X}$ that does. Recall from the beginning of the section that $0 \leq \delta \leq 2$ and since we always have $r \leq d-\delta$ we see that $r \leq l=d-2$ can only be broken if $\delta=1$ and $r=d-1$, or if $\delta=0$ and $r=d$ or $d-1$. Thus adding $D_{1}^{r-(d-2)}$ to $X$ when $r>d-2$ will give an element $\widetilde{X}$ which satisfies our first condition by construction; if $r \leq d-2$ we let $\tilde{X}=X$. Now if $\widetilde{X}$ fails to satisfy $\widetilde{m_{1}}=\widetilde{m_{2}}$ we can simply increase $\widetilde{m_{2}}$ until we reach an element $\bar{X}$ which does. If this element fails to satisfy $2 \overline{m_{1}}-1 \geq \overline{n_{1}}$ we again simply increase $\overline{m_{1}}$ and $\overline{m_{2}}$ and construct an element $\widehat{X}$ which does. Note as previously that increasing $\overline{m_{1}}$ and $\overline{m_{2}}$ in this way may increase $\delta$, thus strengthening the condition $r \leq d-\delta$. However this condition will still hold since $\delta \leq 2$ gives $d-2 \leq d-\delta$ and therefore $r \leq l=d-2$ (which is satisfied by $\widetilde{X}$ and clearly remains satisfied when $\widetilde{m_{1}}, \widetilde{m_{2}} \geq 1$ are increased) implies $r \leq d-\delta$.

We now observe that $f_{D, 2}(\widehat{X})$ is determined by the previous stage and that $X$ can be recovered from $\widehat{X}$ simply by decreasing $\widehat{m_{1}}$ and $\widehat{m_{2}}$ (possibly to zero to reverse to process of creating $\widetilde{X}$ ). We will decrease $\widehat{m_{1}}$ and $\widehat{m_{2}}$ in steps of size one and give the corresponding alterations to make to $f_{D, 2}(\widehat{X})$ at each stage. First though we must introduce a partial order: write $D_{a_{1}} \ldots D_{a_{r}} \preceq D_{b_{1}} \ldots D_{b_{t}}$ if $r \leq t$ and $a_{i} \leq b_{i}$ for all $1 \leq i \leq r$ (here the partial order is simply convenient for stating our algorithm, but it will be of central importance in the future work on sensible functions mentioned at the end of Section 3). We also recall that by convention $D_{0} D_{a}=D_{a}$.

\section{Step one}

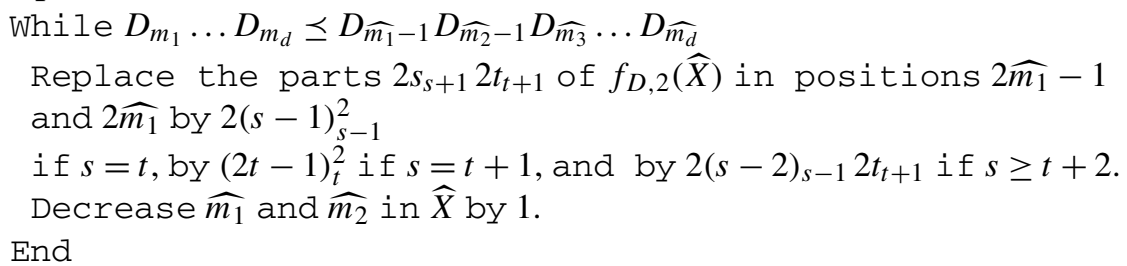

(Step one continues until applying it again would give an element whose $D$-factors preceded the $D$-factors of $X$ in our partial order.) 


\section{Step two}

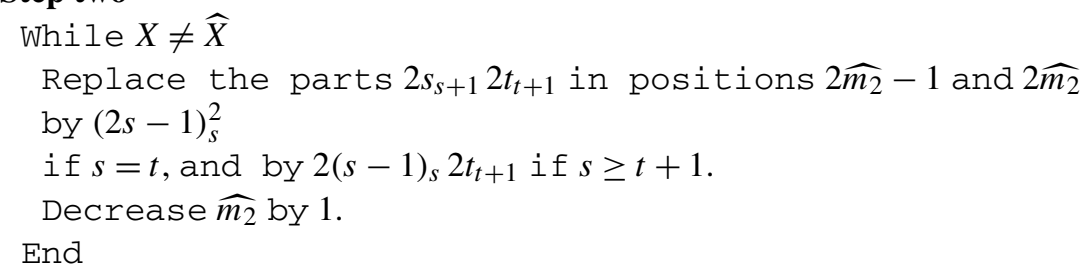

Below we illustrate our algorithm diagrammatically. In each case the first column represents the part in position $2 \widehat{m_{2}}-1$ or $2 \widehat{m_{1}}-1$ as appropriate. Observe that the shapes removed from our symbols here are the same shapes that were added by the algorithm in Theorem 5.1, but upside down. It is unclear if this indicates some 'duality' between the functions $f_{C, 2}$ and $f_{D, 2}$. We shall see that there is no such visual relationship between either of these functions and $f_{B, 2}$.

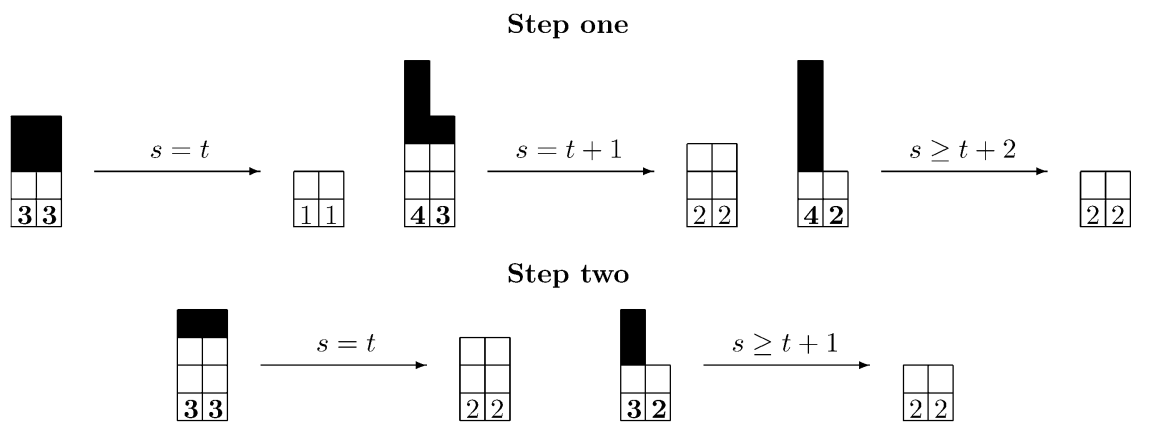

Observe as before that when constructing $\widehat{X}$ we did not require that $\widehat{m_{1}}\left(=\widehat{m_{2}}\right)$ was minimal with respect to $2 \widehat{m_{1}}-1 \geq \widehat{n_{1}}$. So if $X$ satisfies $r \leq d-2, m_{1}=m_{2}$ and $2 m_{1}-1 \geq n_{1}$ and we form $X^{\prime}$ by increasing $m_{1}$ and $m_{2}$ equally, then both $f_{D, 2}(X)$ and $f_{D, 2}\left(X^{\prime}\right)$ will be determined by the formula given in the previous stage, however we could also take $X^{\prime}$ as $\widehat{X}$ and apply the algorithm to $f_{D, 2}\left(X^{\prime}\right)$ to recover $f_{D, 2}(X)$. Therefore we must show that the algorithm is consistent with the formula. So, if $f_{D, 2}(X)$ has $2 m_{1}$ parts and $f_{D, 2}\left(X^{\prime}\right)$ has $2 m_{1}^{\prime}$ parts, then the formula tells us that the parts in positions $2 m_{1}+1$ to $2 m_{1}^{\prime}$ of $f_{D, 2}\left(X^{\prime}\right)$ all have size 2 . Thus to recover $X$ from $X^{\prime}$ we must use Step one of the algorithm $m_{1}^{\prime}-m_{1}$ times and this will decrease the final $2\left(m_{1}^{\prime}-m_{1}\right)$ parts of $f_{D, 2}\left(X^{\prime}\right)$ to zero. Therefore the $f_{D, 2}(X)$ determined by the algorithm is the same as the $f_{D, 2}(X)$ determined by the formula and the function is well-defined.

Next we must check that the symbols our procedure has produced are genuine elements of $\operatorname{uccl}_{2}(D)$. We can see that the descending order of the parts is maintained by an argument similar to that used in the proof of Theorem 5.1; the odd parts in the underlying partitions of our symbols still have even multiplicity, since the symbols of the form $f_{D, 2}(\widehat{X})$ have only even parts and any odd parts created by the algorithm come in pairs; and we do not produce parts of the same size, but with different $\chi$ values. Again this follows by an argument similar to the one used in the proof of Theorem 5.1. 
Finally in this stage we observe that the formula given in the previous stage satisfied properties (A) and (B) and proceding as in the proof of Theorem 5.1 we can show these actually hold for all $X \in \mathrm{Cent}_{2}(D)$.

Stage 3: The values of $2^{\left(X, D_{n}\right)}$.

Let $J$ be the size of the largest Jordan block of $f_{D, 2}(X)$, then as in Theorem 5.1 we have $o_{e}\left(f_{D, 2}(X)\right)=\min \left\{2^{e}: 2^{e} \geq J\right\}$. To match this we must calculate $2^{\left(X, D_{n}\right)}$. Therefore we must find the value of $o_{\min }\left(X, D_{n}\right)$ under all possible conditions. Recall that $r \leq d-\delta, 0 \leq \delta \leq 2$ and that the number of deleted vertices in the extended Dynkin diagram is $d-\delta+1$.

The cases $0 \leq \delta \leq 1$ were dealt with in the proof of Theorem 6.1 , so we suppose that $\delta=2$. Here we delete $d-1$ vertices from the extended Dynkin diagram and since all must have had weight 2 we see that $o_{\min }\left(X, D_{n}\right)=2(d-1)$. So as in the proof of Theorem 6.1, overall we have shown the following:

$$
o_{\min }\left(X, D_{n}\right)= \begin{cases}2 d & \text { if } d=r \\ 2 d-1 & \text { if } d=r+1 \\ 2 d-2 & \text { if } d \geq r+2\end{cases}
$$

Now, if $p \neq 2$ then $2^{\left(X, D_{n}\right)}=2^{i}$ is coprime to $p$ and we can construct $s \in G\left(D_{n}\right)_{p}$ by labeling the vertices so that at most one has label greater than one and the sum of the labels is $2^{\left(X, D_{n}\right)}$.

Now recall from the previous stage that if $r \leq d-2$ then $X=\tilde{X}$. So having formed $\widehat{X}$, to regain $X$ we use the algorithm given in that stage and we note that we shall not decrease $\widehat{m_{1}}$ or $\widehat{m_{2}}$ to 0 , since this would only be done to reverse adding $D_{1}^{r-(d-2)}$. This process never took place when creating $\widehat{X}$ since we started with $X=\widetilde{X}$. Since neither $\widehat{m_{1}}$ nor $\widehat{m_{2}}$ are ever decreased to 0 by the algorithm we also never decrease the parts in positions 1 and 2 . Therefore the largest part of $f_{D, 2}(X)$ is equal to the largest part of $f_{D, 2}(\widehat{X})$ which, by Stage 1 , is $2(d-1)$; hence $o_{e}\left(f_{B, 2}(X)\right)=\min \left\{2^{e}: 2^{e} \geq 2(d-1)\right\}$. Since in this case we also have $o_{\min }\left(X, D_{n}\right)=2 d-2$ it follows that $2^{\left(X, D_{n}\right)}=o_{e}\left(f_{B, 2}(X)\right)$.

Thus the only cases left to check are when $d=r$ or $r+1$. This time when we form $\tilde{X}$ we get $\widetilde{d}=r+2=d+2$ or $d+1$ respectively. Now when we form $\widehat{X}$ we don't change the number of $D$-factors, so $\widehat{d}=\widetilde{d}$. Therefore from Stage 1 we see that the first two parts of $f_{D, 2}(\widehat{X})$ are

$$
2(\widehat{l}+1)_{\widehat{l}+2} 2(r+1)_{r+2}=2(\widehat{d}-1)_{\widehat{d}} 2(r+1)_{r+2}= \begin{cases}2(d+1)_{d+2}^{2} & \text { if } d=r \\ 2 d_{d+1}^{2} & \text { if } d=r+1\end{cases}
$$

Now to recover $X$ from $\widehat{X}$ we will decrease both $\widehat{m_{1}}$ and $\widehat{m_{2}}$ to 0 if $d$ was $r$, and just $\widehat{m_{2}}$ to 0 if $d$ was $r+1$. To match this we will, in particular, apply Step one or Step two respectively to the parts in positions 1 and 2 .

So if $d=r$ we have $J=2 d=o_{\min }\left(X, D_{n}\right)$, and if $d=r+1$ we have $J=2 d-$ $1=o_{\min }\left(X, D_{n}\right)$. In either case $2^{\left(X, D_{n}\right)}=o_{e}\left(f_{B, 2}(X)\right)$, so property $(\mathrm{C}+)$ has been demonstrated and the proof is complete. 


\section{Type $B$}

In this section we will deal with groups of type $B$. We begin as before with the extended Dynkin diagram of type $\widetilde{B_{n}}$, together with the weights obtained from the highest root.

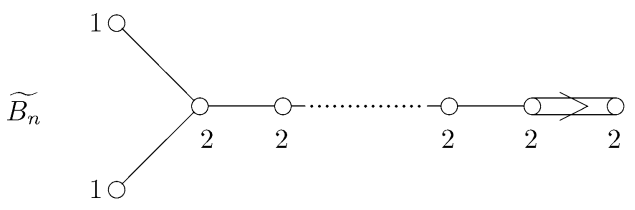

Again we should make clear our conventions regarding groups with small rank. $B_{2}$ and $B_{1}$ can be defined and are isomorphic to $C_{2}$ and $A_{1}$ respectively, but as subgroups of a larger $B_{n}$ we know that the $B_{2}$ s form a single conjugacy class, whereas $B_{1}$ and $A_{1}$ are not conjugate and hence are viewed as different. We take $B_{0}$ to be the trivial group.

Once again Algorithm 2.2 allows us to list the $\sim_{t}$ classes of connected centralizers of semisimple elements. As with type $D$ we must observe that $T_{l}$ can also be written as $D_{1}^{l}$. Although we could use either notation when constructing $f_{B, q}$ for odd $q$, it will be more convenient to write $D_{1}^{l}$ when $q=2$. Therefore in the interests of consistency we will use this notation for the remainder of the section. Thus the connected centralizers of semisimple elements in type $B$ have the form $X=A_{n_{1}} \ldots A_{n_{r}} B_{w} D_{m_{1}} \ldots D_{m_{d}}$, where $n_{1} \geq \ldots \geq n_{r}$ and $m_{1} \geq \ldots \geq m_{d}$. Algorithm 2.2 also shows that the number of $D$-factors with rank at least 2 (denoted $\delta$ ) is less than or equal to 1 and that the number of $A$-factors $(r)$ can be no more than the number of deleted vertices minus one, which equals the dimension of the central torus, i.e. $d-\delta$.

Now observe that all primes are admissible with respect to any connected centralizer with $\delta=0$, but for connected centralizers with $\delta=1$ only 2 is admissible. This follows since if $\delta=0$ then when obtaining the connected centralizer from Algorithm 2.2 at least one deleted vertex has weight 1 ; we can label that vertex such that the weighted sum of the labels is $q^{i}$ for some $i$. If all deleted vertices have weight 2 then the weighted sum must be even and so can only be a prime power if the prime is two.

Thus all the sets $\operatorname{Cent}_{q}(B)$, for odd $q$, are the same; they all equal

$$
\left\{A_{n_{1}} \ldots A_{n_{r}} B_{w} D_{1}^{l}: n_{1}, \ldots, n_{r}, w, l \in \mathbb{N} \cup\{0\}, \text { where } n_{1} \geq \ldots \geq n_{r}, \text { and } r \leq l\right\}
$$

The set $\operatorname{Cent}_{2}(B)$ equals

$$
\begin{aligned}
& \left\{A_{n_{1}} \ldots A_{n_{r}} B_{w} D_{m_{1}} D_{1}^{l}: n_{1}, \ldots, n_{r}, w, m_{1}, l \in \mathbb{N} \cup\{0\},\right. \\
& \text { where } \left.n_{1} \geq \ldots \geq n_{r}, r \leq l, \text { and } m_{1} \geq 0\right\}
\end{aligned}
$$


Next we consider unipotent conjugacy classes in type $B_{n}$ over odd characteristic $q$ (postponing the treatment of characteristic 2 until later). As before, from [3] we see that these are parameterized by symbols $p_{1} \chi_{1}{ }_{1} \cdots p_{z} \chi_{z}$, where $p_{1}{ }^{m_{1}} \cdots p_{z}{ }^{m_{z}}$ is a partition of $2 n+1$ given by the Jordan structure on the natural module, each even part has even multiplicity and $\chi: p_{j} \mapsto \chi_{j}$ is a function from the parts of $p_{1}{ }^{m_{1}} \cdots p_{z}{ }^{m_{z}}$ to the natural numbers satisfying

$$
\chi(p)= \begin{cases}\frac{1}{2}(p+1) & \text { if } p \text { is odd } \\ \frac{1}{2} p & \text { if } p \text { is even. }\end{cases}
$$

By identifying members $\mathbf{p} \in \operatorname{uccl}_{q}(B)$ with the appropriate symbol we again get a bijective representation and we will use this identification from now on. Now by definition $r(\mathbf{p})=n=\frac{1}{2}(|\mathbf{p}|-1)$, from [3] we see that if $\mathbf{p}=p_{1}{ }_{1}^{m_{1}} \cdots p_{z} \chi_{z}$, then

$$
\text { c.dim } \mathbf{p}=\sum_{i=1}^{z}\left(i p_{i}-\chi\left(p_{i}\right)\right)
$$

and $o_{e}(\mathbf{p})=\min \left\{q^{i} ; q^{i} \geq p_{1}\right\}$. We repeat the now standard warning to avoid ambiguity by always stating $q$ when talking about $o_{e}(\mathbf{p})$. Now with all the notation in place we obtain the following result.

Theorem 7.1 For each odd prime $q$, there exists a function $f_{B, q}: \operatorname{Cent}_{q}(B) \rightarrow$ $\operatorname{uccl}_{q}(B)$ satisfying properties $(A),(B)$ and $(C+)$. Furthermore, if $p \neq q$ then there exists a semisimple element $s \in G\left(B_{n}\right)_{p}$ such that $C_{G\left(B_{n}\right)_{p}}(s) \sim_{t} X$ and $o(s)=q^{\left(X, B_{n}\right)}$.

Proof The reader knows the drill by now.

Stage 1: Defining $f_{B, q}\left(A_{n_{1}} \ldots A_{n_{r}} B_{w} D_{1}^{l}\right)$ when $2 w \geq n_{1} \geq \cdots \geq n_{r}$.

If $\mathbf{n}=\left(n_{1}, \ldots, n_{r}\right)$, where $2 w \geq n_{1} \geq \cdots \geq n_{r}$ then we let

$$
\begin{aligned}
f_{B, q}\left(A_{n_{1}} \ldots A_{n_{r}} B_{w} D_{1}^{l}\right) & =2\left(l+1,(2 w, \mathbf{n})^{*}\right)-1 \\
& =(2 l+1)_{l+1}(2 r+1)_{r+1}^{n_{r}}(2 r-1)_{r}^{n_{r-1}-n_{r}} \cdots 1_{1}^{2 w-n_{1}} .
\end{aligned}
$$

It is easily seen that the two partitions are equal and properties (A) and (B) may be proven as in previous sections.

Stage 2: Defining $f_{B, q}(X)$ for arbitrary $X$.

Here we define the image $f_{B, q}(X)$ of an arbitrary connected centralizer $X$. If $X$ satisfies $2 w \geq n_{1}$ then the image has already been determined, if not we can simply increase $w$ and construct a connected centralizer $\widehat{X}$ which does. We now observe that $f_{B, q}(\widehat{X})$ is determined by the previous stage and that $X$ can be recovered from $\widehat{X}$ simply by decreasing $\widehat{w}$. We will decrease $\widehat{w}$ in steps of size one and give the corresponding alterations to make to $f_{B, q}(\widehat{X})$ at each stage. We proceed as follows. 


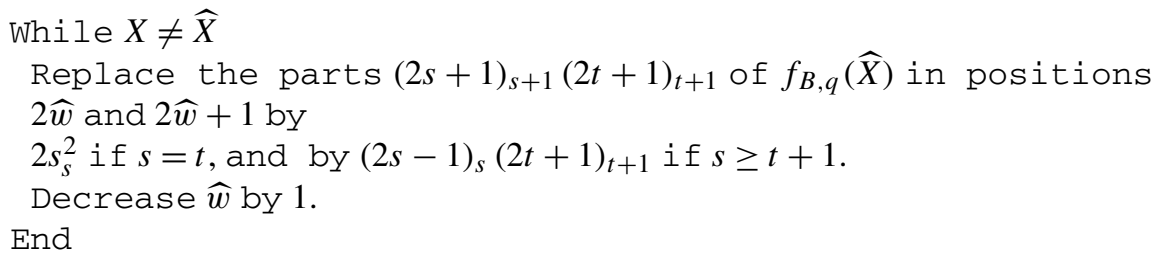

Below we illustrate our algorithm diagrammatically. In both cases the first column represents the part in position $2 \widehat{w}$.

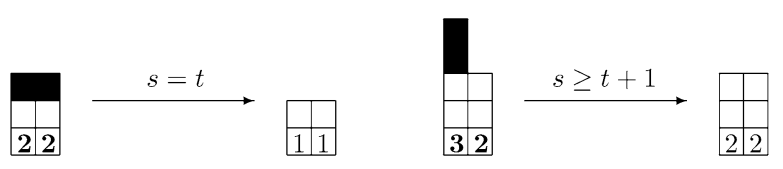

Note that, as in the previous section, $\widehat{w}$ need not be minimal. However using the same arguments as in the previous section we can show that the $f_{B, q}(X)$ determined by the algorithm (using any $\widehat{X}$ ) equals the $f_{B, q}(X)$ determined by the formula. So our definitions are not inconsistent.

Now before proceeding any further we must check that the symbols our procedure has produced are genuine elements of $\operatorname{uccl}_{q}(B)$. As before what we need to check is that the descending order of the parts is maintained, that the even parts in the underlying partitions of our symbols still have even multiplicity and that properties (A) and (B) hold in general. None of this is difficult or significantly different to the corresponding checks in previous cases.

Stage 3: The values of $q^{\left(X, B_{n}\right)}$.

Observe that the algorithm given in the previous stage will never decrease the largest part of the partition when producing $f_{B, q}(X)$. So we will always have $o_{e}\left(f_{B, q}(X)\right)=\min \left\{q^{e}: q^{e} \geq 2 l+1\right\}$. To match this we must calculate $q^{\left(X, B_{n}\right)}$. Therefore we must find the value of $o_{\min }\left(X, B_{n}\right)$ under all possible conditions. Recall that $r \leq l$ and that the number of deleted vertices in the extended Dynkin diagram after applying Algorithm 2.2 is $l+1$.

So if $r=l$ then the number of deleted vertices is one more than the number of subgraphs of type $A$ left. We deduce that only one of the vertices of weight 1 has been deleted and so $o_{\min }\left(X, B_{n}\right)=2 l+1$, as when the labels ${ }_{0}^{1}{ }^{00100100}$ give a semisimple element of order 5 with a connected centralizer of type $A_{3} A_{2} B_{2} D_{1}^{2}$. Whereas if $r \leq l-1$ then the number of deleted vertices is at least two more than the number of subgraphs of type $A$ left. Hence both of the vertices of weight 1 have been deleted (as in Theorem 6.1 we will use as many weight 1 vertices as we can since we are looking for the semisimple element with the smallest possible order) and we get $o_{\min }\left(X, B_{n}\right)=2(l-1)+1+1=2 l$, as when the labels ${ }^{1}{ }^{00100100}$ give a semisimple element of order 6 with a connected centralizer of type $A_{2}^{2} B_{2} D_{1}^{3}$. Now we have shown that $q^{\left(X, B_{n}\right)}=\min \left\{q^{e}: q^{e} \geq o_{\min }\left(X, B_{n}\right)\right\}$, where $o_{\min }\left(X, B_{n}\right)=2 l+1$ or $2 l$. However since $q$ is odd and $2 l$ is even we must have $q^{\left(X, B_{n}\right)}=\min \left\{q^{e}: q^{e} \geq 2 l+1\right\}=o_{e}\left(f_{B, q}(X)\right)$. Thus property $(\mathrm{C}+)$ holds. 
Finally, if $p \neq q$ then $q^{\left(X, B_{n}\right)}=q^{i}$ is coprime to $p$ and we can construct $s \in$ $G\left(B_{n}\right)_{p}$ by labeling the vertices so that at most one has label greater than one and the sum of the labels is $q^{\left(X, B_{n}\right)}$. This can always be done since we see by the above that at least one vertex of weight 1 will be deleted.

We can now consider unipotent conjugacy classes in type $B_{n}$ over characteristic 2 . Following [3], these are again parameterized by symbols $\mathbf{p}=p_{1}{ }_{1}^{t_{1}} \cdots p_{z}{ }_{z}^{t_{z}}$ (again giving a bijective representation and an identification). Here $p_{1}{ }^{t_{1}} \cdots p_{z} t_{z}$ is a partition of $2 n+1$ given by the Jordan structure, where each odd part greater than 1 has even multiplicity. Note that if all odd parts had even multiplicity the sum of the parts would be even; therefore since we want a partition of $2 n+1$ we must have the part 1 occurring with odd multiplicity, in particular the final part of a symbol will always be 1 . The map $\chi: p_{j} \mapsto \chi_{j}$ from the parts of $p_{1}{ }^{t_{1}} \cdots p_{z}{ }^{t_{z}}$ to the natural numbers satisfies the following rule:

$$
\chi(p)= \begin{cases}\frac{1}{2}(p+1) & \text { if } p \text { is odd; } \\ \frac{1}{2}(p+2) & \text { if } p \text { is even and has odd multiplicity; } \\ \frac{1}{2} p \text { or } \frac{1}{2}(p+2) & \text { if } p \text { is even and has even multiplicity. }\end{cases}
$$

For the last time we note that $r(\mathbf{p})=n=\frac{1}{2}(|\mathbf{p}|-1)$ and from [3] we see that if $\mathbf{p}=p_{1 \chi_{1}}^{m_{1}} \cdots p_{z \chi_{z}}^{m_{z}}$, then

$$
\operatorname{cdim} \mathbf{p}=\sum_{i=1}^{z}\left(i p_{i}-\chi\left(p_{i}\right)\right)
$$

and $o_{e}(\mathbf{p})=\min \left\{q^{i} ; q^{i} \geq p_{1}\right\}$. We also repeat the warning to avoid ambiguity by always stating $q$ when talking about $o_{e}(\mathbf{p})$ for the last time.

Theorem 7.2 There exists a function $f_{B, 2}: \operatorname{Cent}_{2}(B) \rightarrow \operatorname{uccl}_{2}(B)$ satisfying properties $(A)$ and $(B)$. Furthermore, if $X \in \mathrm{Cent}_{2}(B)$, then $2^{\left(X, B_{n}\right)}=o_{e}\left(f_{B, 2}(X)\right)$ unless $w=0$ and $r=d=2^{i}$ for some $i$. In this case we have $2^{\left(X, B_{n}\right)}=2 . o_{e}\left(f_{B, 2}(X)\right)$. Finally if $p \neq 2$ then there exists a semisimple element $s \in G\left(B_{n}\right)_{p}$ such that $C_{G\left(B_{n}\right)_{p}}(s) \sim_{t} X$ and $o(s)=2^{\left(X, B_{n}\right)}$.

Proof This is the final proof of this type. However it is also the most complex, so we will spend a little more time on some of the details than has become the norm.

Stage 1: Defining $f_{B, 2}\left(A_{n_{1}} \ldots A_{n_{r}} B_{w} D_{w+1} D_{1}^{l}\right)$ when $r \leq l$ and $2 w \geq n_{1} \geq \cdots \geq n_{r}$.

First note that $2 w \geq 0$ implies that $w+1 \geq 1$ and so $d$ (the number of $D$ factors) is $l+1$. Now set $\mathbf{n}=\left(n_{1}, \ldots, n_{r}\right)$, then when $2 w \geq n_{1} \geq \cdots \geq n_{r}$ and $r \leq l$ we have

$$
\begin{gathered}
f_{B, 2}\left(A_{n_{1}} \ldots A_{n_{r}} B_{w} D_{w+1} D_{1}^{l}\right)=\left(2\left(l+1,(2 w, \mathbf{n})^{*}\right), 1\right) \\
=2(l+1)_{l+2} 2(r+1)_{r+2}^{n_{r}} 2 r_{r+1}^{n_{r-1}-n_{r}} \\
\ldots 4_{3}^{n_{1}-n_{2}} 2_{2}^{2 w-n_{1}} 1_{1} .
\end{gathered}
$$


It is easily seen that the two partitions are equal and properties (A) and (B) are proven as before.

Stage 2: Defining $f_{B, 2}(X)$ when $X$ is arbitrary.

Take $X \in \operatorname{Cent}_{2}(B)$ : if it satisfies the conditions of the previous stage then the image has already been defined, if not we will again construct an element $\widehat{X}$ that does. Recall from the beginning of the section that $0 \leq \delta \leq 1$ and since we always have $r \leq d-\delta$ we see that $r \leq l=d-1$ can only be broken if $\delta=0$ and $r=d$. Thus adding $D_{1}$ to $X$ when $r>d-1$ will give an element $\tilde{X}$ which satisfies our first condition by construction; if $r \leq d-1$ we let $\widetilde{X}=X$. Now if $\widetilde{X}$ fails to satisfy $\widetilde{m_{1}}=\widetilde{w}+1$ we can simply increase $\widetilde{m_{1}}$ or $\widetilde{w}$ until we reach an element $\bar{X}$ which does. If this element fails to satisfy $2 \bar{w} \geq \overline{n_{1}}$ we again simply increase $\bar{w}$ and construct an element $\widehat{X}$ which does. Note as previously that increasing $\overline{m_{1}}=\bar{w}+1$ in this way may increase $\delta$, thus strengthening the condition $r \leq d-\delta$. However this condition will still hold since $\delta \leq 1$ gives $d-1 \leq d-\delta$ and therefore $r \leq l=d-1$ (which is satisfied by $\widetilde{X}$ and clearly remains satisfied when $\widetilde{m_{1}}$ is increased) implies $r \leq d-\delta$.

We now observe that $f_{B, 2}(\widehat{X})$ is determined by the previous stage and that $X$ can be recovered from $\widehat{X}$ simply by decreasing $\widehat{w}$ and $\widehat{m_{1}}$ (possibly to zero to reverse to process of creating $\widetilde{X}$ ). We will decrease $\widehat{w}$ and $\widehat{m_{1}}$ in steps of size one and give the corresponding alterations to make to $f_{B, 2}(\widehat{X})$ at each stage. Now let $\chi(b)=\frac{1}{2} b+1$ if $b$ is even, and $\frac{1}{2}(b+1)$ if $b$ is odd. We then proceed as follows.

\section{Step one}

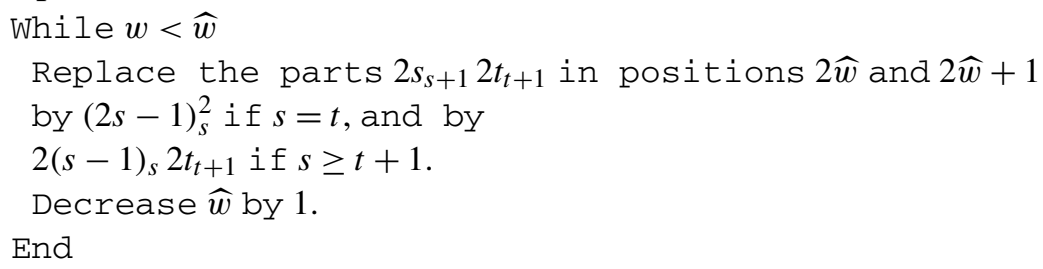

\section{Step two}

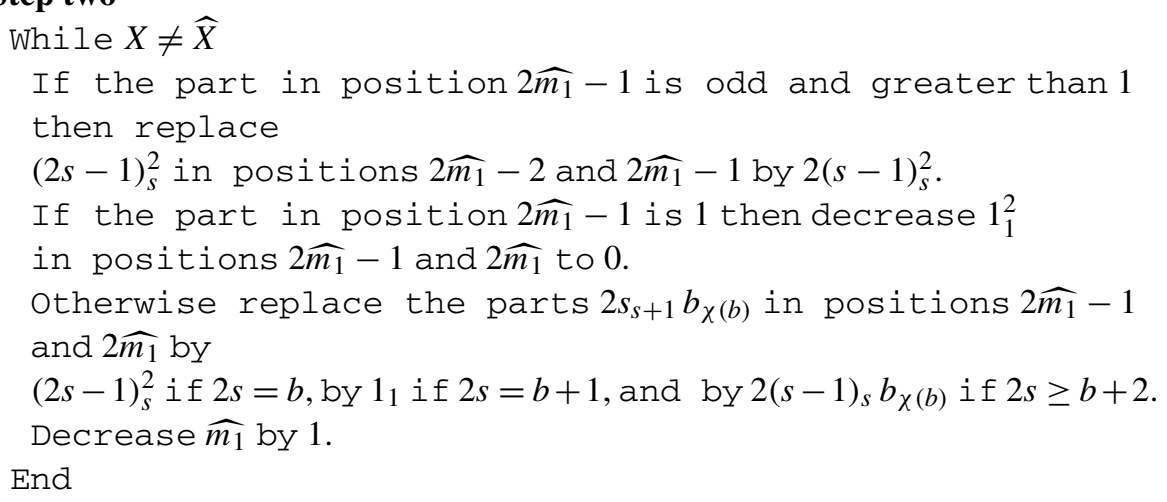

Below we illustrate our algorithm diagrammatically. In each case the upward arrow $(\uparrow)$ points to the part in position $2 \widehat{w}$ or $2 \widehat{m_{1}}-1$ as appropriate to the step. As the reader can see, this algorithm is slightly more complex than those seen previously, so we will go into a little more detail than we have in the last few cases. 
Step one

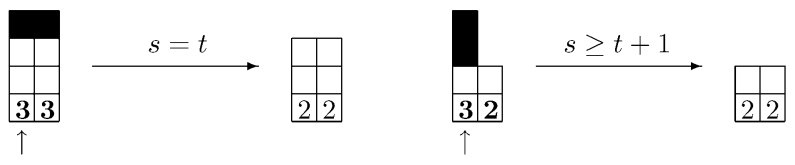

Step two

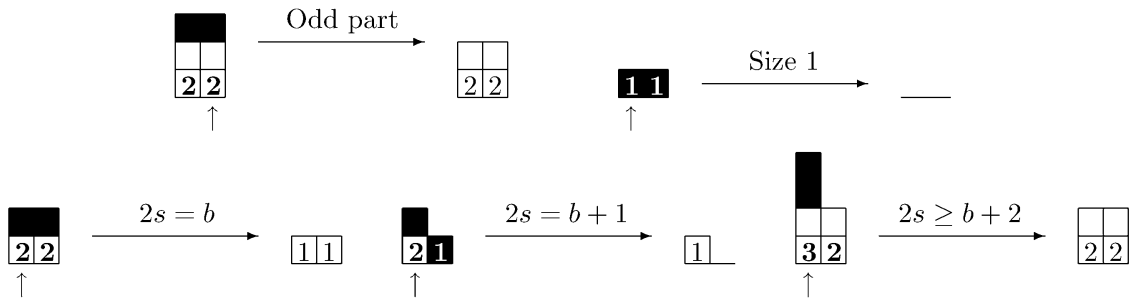

Now observe that when constructing $\widehat{X}$ we did not require that $\widehat{m_{1}}(=\widehat{w}+1)$ was minimal with respect to $2 \widehat{w} \geq \widehat{n_{1}}$. So if $X$ satisfies $d \geq r+1, m_{1}=w+1$ and $2 w \geq n_{1}$ and we form $X^{\prime}$ by increasing $m_{1}$ and $w$, then both $f_{B, 2}(X)$ and $f_{B, 2}\left(X^{\prime}\right)$ will be determined by the formula given in the previous stage, however we could also take $X^{\prime}$ as $\widehat{X}$ and apply the algorithm to $f_{B, 2}\left(X^{\prime}\right)$ to recover $f_{B, 2}(X)$. Therefore we must show that the algorithm is consistent with the formula. So, if $f_{B, 2}(X)$ has $2 m_{1}$ parts and $f_{B, 2}\left(X^{\prime}\right)$ has $2 m_{1}^{\prime}$ parts, then the formula tells us that the parts in positions $2 m_{1}$ to $2 m_{1}^{\prime}-1$ of $f_{B, 2}\left(X^{\prime}\right)$ all have size 2 and the part in position $2 m_{1}^{\prime}$ has size 1 . Thus to recover $X$ from $X^{\prime}$ we must use Step one of the algorithm $m_{1}^{\prime}-m_{1}$ times, followed by Step two $m_{1}^{\prime}-m_{1}$ times. After using Step one $m_{1}^{\prime}-m_{1}$ times the final $2\left(m_{1}^{\prime}-m_{1}\right)+1$ parts of $f_{B, 2}\left(X^{\prime}\right)$ will have size 1 . Then applying Step two $m_{1}^{\prime}-m_{1}$ times will decrease the final $2\left(m_{1}^{\prime}-m_{1}\right)$ parts to 0 . Therefore the $f_{B, 2}(X)$ determined by the algorithm equals the $f_{B, 2}(X)$ determined by the formula.

The next thing to do is identify and verify some implicit claims made in Step two of our algorithm. Having applied Step one, if the part in position $2 \widehat{m_{1}}-1$ is odd and greater than 1 , we claim that the part in position $2 \widehat{m_{1}}-2$ has the same size. Also, since there is no part in position zero we claim that after Step one the part in position 1 is not odd. This second claim is easily seen to be true since in the symbol $f_{B, 2}(\widehat{X})$ the first part must be even $(d \geq r+1 \geq 1$ and by the formula given in the previous stage the first part of $f_{B, 2}(\widehat{X})$ is $\left.2 d\right)$. So since Step one of the algorithm does not act on the first part (as with $f_{B, q}$ for odd $q$ ) we cannot have an odd first part for Step two to act upon. Now returning to the first claim we see that since $f_{B, 2}(\widehat{X})$ had no odd parts greater than one, any such parts acted upon by Step two of our algorithm must have been created during Step one. From Step one we then see that if the part in position $2 \widehat{w}+1$ is made odd then the part in position $2 \widehat{w}$ is also made odd and will have the same size. The claim follows.

We also should prove that if the part in position $2 \widehat{m_{1}}-1$ has size 1 after Step one, then so does the part in position $2 \widehat{m_{1}}$. To see this we will first observe that the descending order of the parts is maintained by Step one of the above algorithm. Clearly $f_{B, 2}(\widehat{X})$ had parts in descending order of size and the descending order of the parts in 
positions $2 \widehat{w}$ and $2 \widehat{w}+1$ is obviously preserved in Step one. So since the part in position $2 w$ is decreased by at least 1 and the part in position $2 w+1$ is decreased by at most 1 during Step one, the descending order of the parts is preserved in all positions during that step. An immediate consequences of this is that Step one doesn't change the number of parts (since it doesn't decrease the final part of $f_{B, 2}(\widehat{X})$ ). Now if the part in position $2 \widehat{m_{1}}-1$ has size 1 after Step one, then the part in position $2 \widehat{m_{1}}$ must have size one or zero. However in the latter case the part in position $2 \widehat{m_{1}}-1$ would be the final part, which is impossible since $f_{B, 2}(\widehat{X})$ had an even number, $2(\widehat{w}+1)$, of parts; our original claim follows.

Our final implicit claim is that if, after Step one, the part in position $2 \widehat{m_{1}}-1$ is even and has size 1 greater than the part in position $2 \widehat{m_{1}}$, then these parts must be $2_{2} 1_{1}$. Otherwise property (A) would be broken by replacing the parts by $1_{1}$. So when applying Step two we claim that if the part in position $2 \widehat{m_{1}}-1$ is even and greater than 2 after Step one, then the part in position $2 \widehat{m_{1}}$ does not have size 1 less than $i t$. We only need to consider the case that the part in position $2 \widehat{m_{1}}$ is odd and greater than 1 after Step one: then the part in position $2 \widehat{m_{1}}+1$ must have the same size and applying Step two to match decreasing $\widehat{m_{1}}+1$ to $\widehat{m_{1}}$ will decrease both parts by 1 . So when applying Step two to match decreasing $\widehat{m_{1}}$ to $\widehat{m_{1}}-1$ we will have an even part in position $2 \widehat{m_{1}}$. The claim follows.

An example of the algorithm at work is illustrated below. The first row shows the action of Step one and the second row continues with the action of Step two.
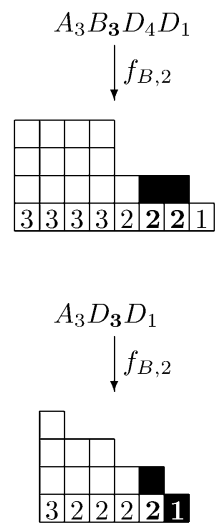
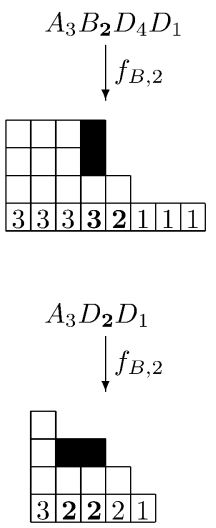
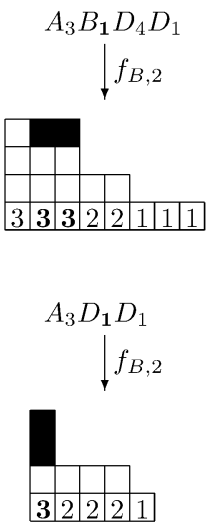

$A_{3} D_{4} D_{1}$
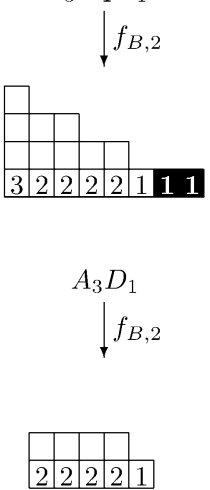

We will now complete our check that the algorithm maintains the descending order of the parts. For Step two we must show that the part $x_{j}$ following a part to be decreased is never greater than the size of that part after the decrease. If the parts in positions $2 \widehat{m_{1}}-1$ and $2 \widehat{m_{1}}$ have the form $2 s_{s+1} b_{\chi(b)}$, where $2 s \geq b+2$ then this will clearly be true. If the parts in positions $2 \widehat{m_{1}}-1$ and $2 \widehat{m_{1}}$ have the form $21_{1}$ or $1_{1}^{2}$ then, since the descending order of the parts is certainly maintained before Step two is applied, we see that the part in position $2 \widehat{m_{1}}+1$ must have had size 1 or 0 after Step one. If it had size 1 then when Step two was matching decreasing $\widehat{m_{1}}+1$ to $\widehat{m_{1}}$ it would have decreased it to 0 ; either way the descending order is maintained. If the parts in positions $2 \widehat{m_{1}}-1$ and $2 \widehat{m_{1}}$ have the form $2 s_{s+1}^{2}$ then, again since the descending order of the parts is certainly maintained before Step two is applied, we 
see that the part in position $2 \widehat{m_{1}}+1$ must have had size no greater than $2 s$ after Step one. If it had size $2 s$ then when Step two was matching decreasing $\widehat{m_{1}}+1$ to $\widehat{m_{1}}$ it would have decreased it and the descending order of the parts is preserved. Similarly, if the parts in positions $2 \widehat{m_{1}}-2$ and $2 \widehat{m_{1}}-1$ have the form $(2 s+1)_{s+1}^{2}$ where $s>1$ then, since the descending order of the parts is maintained before Step two is applied, we see that the part in position $2 \widehat{m_{1}}$ must have had size no greater than $2 s+1$ after Step one. If it had size $2 s+1>3$ then since no such parts existed in $f_{B, 2}(\widehat{X})$ it must have been created during Step one. Therefore the part in position $2 \widehat{m_{1}}+1$ must also have had size $2 s+1$ after Step one. So when Step two was matching decreasing $\widehat{m_{1}}+1$ to $\widehat{m_{1}}$ it would have decreased the parts in positions $2 \widehat{m_{1}}$ and $2 \widehat{m_{1}}+1$ and the descending order of the parts is preserved. The preservation of the descending order of the parts by Step two of our algorithm follows.

To completely satisfy ourselves that the symbols our procedure has produced are genuine elements of $\operatorname{uccl}_{2}(B)$ we must check two more things. Firstly, the multiplicities of the odd parts in the underlying partitions of our symbols still have the required parity since the symbols of the form $f_{B, 2}(\widehat{X})$ only had one odd part of size 1 and additional odd parts are created or destroyed by the algorithm in pairs. Secondly, we do not produce parts of the same size, but with different $\chi$-values since all $\chi$-values always take the largest possible values.

Finally in this stage we observe that the formula given in the previous stage satisfied properties (A) and (B) and proceding as in the proof of Theorem 5.1 we can show these actually hold for all $X \in \mathrm{Cent}_{2}(B)$.

Stage 3: The values of $2^{\left(X, B_{n}\right)}$.

Let $J$ be the size of the largest Jordan block of $f_{B, 2}(X)$, then as in Theorem 5.1 we have $o_{e}\left(f_{B, 2}(X)\right)=\min \left\{2^{e}: 2^{e} \geq J\right\}$. To match this we must calculate $2^{\left(X, B_{n}\right)}$. Therefore we must find the value of $o_{\min }\left(X, B_{n}\right)$ under all possible conditions. Recall that $r \leq d-\delta, 0 \leq \delta \leq 1$ and that the number of deleted vertices in the extended Dynkin diagram is $d-\delta+1$.

First suppose that $\delta=0$ (so there is no $D$-factor of rank greater than one): here we delete $d+1$ vertices from the extended Dynkin diagram and consider two subcases. If $d=r$ then the number of vertices deleted after applying Algorithm 2.2 is one more than the number of subgraphs of type $A$ left. We deduce that only one vertex of weight 1 has been deleted and so $o_{\min }\left(X, B_{n}\right)=2 d+1$. For example, the labels $0^{1}{ }^{00100100}$ give a semisimple element of order 5 with a connected centralizer of type $A_{3} A_{2} B_{2} D_{1}^{2}$. Whereas if $r \leq d-1$ then the number of vertices deleted after applying Algorithm 2.2 is at least two more than the number of subgraphs of type $A$ left. We deduce that both vertices of weight 1 have been deleted (as in previous proofs we remind the reader that we use as many weight 1 vertices as we can since we are looking for the semisimple element with the smallest possible order) and $o_{\min }\left(X, B_{n}\right)=2(d-1)+1+1=2 d$, as when the labels ${ }^{1}{ }^{00100100}$ give a semisimple element of order 6 with a connected centralizer of type $A_{2}^{2} B_{2} D_{1}^{3}$.

Now let $\delta=1$ (so there is a $D$-factor of rank greater than one): here we delete $d$ vertices from the extended Dynkin diagram and since no vertices of weight 1 have been deleted we get $o_{\min }\left(X, B_{n}\right)=2 d$. Therefore overall we have shown the following: 


$$
o_{\min }\left(X, B_{n}\right)= \begin{cases}2 d+1 & \text { if } d=r \\ 2 d & \text { if } d \geq r+1 .\end{cases}
$$

Now, if $p \neq 2$ then $2^{\left(X, B_{n}\right)}=2^{i}$ is coprime to $p$ and we can construct $s \in G\left(B_{n}\right)_{p}$ by labeling the vertices so that at most one has label greater than one and the sum of the labels is $2^{\left(X, B_{n}\right)}$.

Now recall from the previous stage that if $r \leq d-1$ then $X=\tilde{X}$. So having formed $\widehat{X}$, to regain $X$ we use the algorithm given in that stage and we note that we shall not decrease $\widehat{m_{1}}$ to 0 , since this would only be done to reverse adding a $D_{1}$. This process never took place when creating $\widehat{X}$ since we started with $X=\widetilde{X}$. Now it was observed when verifying the first of implicit assumptions about Step two that Step one of our algorithm does not decrease the part of $f_{B, 2}(\widehat{X})$ in position 1 . Since $\widehat{m_{1}}$ is never decreased to 0 by the algorithm we also see that Step two never decreases the parts in positions 1 and 2 . Therefore the largest part of $f_{B, 2}(X)$ is equal to the largest part of $f_{B, 2}(\widehat{X})$ which, by Stage 1 , is $2(l+1)=2 d$. Thus if $r \leq d-1$ we have $o_{e}\left(f_{B, 2}(X)\right)=\min \left\{2^{e}: 2^{e} \geq 2 d\right\}$, which equals $2^{\left(X, B_{n}\right)}$ by the previous paragraph.

Thus the only case left to check is when $d=r$. If $r=d=0$ then $X=B_{w}$ and so $\widehat{X}=B_{w} D_{w+1}, f_{B, 2}(\widehat{X})=2_{2}^{2 w+1} 1_{1}$ and $f_{B, 2}(X)=1_{1}^{2 w+1}$. Hence $J=1=2 d+1$, which equals $o_{\min }\left(X, B_{n}\right)$ by the above, and we get $q^{\left(X, B_{n}\right)}=o_{e}\left(f_{B, 2}(X)\right)$. Therefore for the remainder of the proof we will assume $d=r \neq 0$. So observe that when we form $\widetilde{X}$ we get $\widetilde{d}=r+1=d+1$. Now when we form $\widehat{X}$ we don't change the number of $D$-factors so $\widehat{d}=\widetilde{d}=d+1$. Therefore from Stage 1 we see that the first two parts of $f_{B, 2}(\widehat{X})$ are $2 \widehat{d}_{\widehat{d}+1} 2(r+1)_{r+2}=2(d+1)_{d+2}^{2}$. Now when recovering $X$ from $\widehat{X}$, Step one of our algorithm will never act on the first part of our symbol, and will only act on the second part if we had $w=0$ in $X$. Suppose this was not the case, then proceed to Step two. Here we will decrease $\widehat{m_{1}}$ to 0 and to match this we will, in particular, replace the parts $2(d+1)_{d+2}^{2}$ in positions 1 and 2 by $(2 d+1)_{d+1}^{2}$. Therefore $J=2 d+1$, which again equals $o_{\min }\left(X, B_{n}\right)$ by the above, and we get $q^{\left(X, B_{n}\right)}=o_{e}\left(f_{B, 2}(X)\right)$.

Now suppose that $d=r \neq 0$, but we did have $w=0$ in $X$; from above we know that the first two parts of $f_{B, 2}(\widehat{X})$ have the form $2(d+1)_{d+2}^{2}$. Now recall that $\widehat{X}=$ $A_{n_{1}} \ldots A_{n_{r}} B_{\widehat{w}} D_{\widehat{w}+1} D_{1}^{l}$, where $2 \widehat{w} \geq n_{1} \geq \ldots \geq n_{r}$. Since $r \neq 0$ we have $A$-factors of non-zero rank and hence $\widehat{w} \geq 1$ and $f_{B, 2}(\widehat{X})$ has $2(\widehat{w}+1) \geq 4$ parts. Now if the parts in positions 2 and 3 are equal, then we see that Step one will leave the first three parts with the form $2(d+1)_{d+2}(2 d+1)_{d+1}^{2}$. Applying Step two we must decrease $\widehat{m_{1}}$ to 0 as above and to match the decrease of $\widehat{m_{1}}$ from 2 to 1 we will transform the first three parts into $2(d+1)_{d+2} 2 d_{d+1}^{2}(d \geq 1$ implies that the third part is odd and greater than one). Matching the decrease from 1 to 0 we then get $2 d_{d+1}^{3}$ and $J=2 d$. Alternatively, if the parts in positions 2 and 3 of $f_{B, 2}(\widehat{X})$ are not equal, then we see that Step one will leave the first three parts with the form $2(d+1)_{d+2} 2 d_{d+1} 2 b_{b+1}$, where $d \geq b$. This time applying Step two to match the decrease of $\widehat{m_{1}}$ from 2 to 1 will not affect the second part since the third part is even. So matching the decrease from 1 to 0 we get the first two parts of $f_{B, 2}(X)$ of the form $2 d_{d+1}^{2}$. So again we have $J=2 d$ and in either subcase we get $2^{\left(X, B_{n}\right)}=\min \left\{2^{e}: 2^{e} \geq 2 d+1\right\}$, which equals $\min \left\{2^{e}: 2^{e} \geq 2 d\right\}=o_{e}\left(f_{B, 2}(X)\right)$ unless $d=2^{i}$ for some $i$. In this case we get $2^{\left(X, B_{n}\right)}=2 . o_{e}\left(f_{B, 2}(X)\right)$. 


\section{The proof of Theorem 3}

In this final section we will summarize what we have proven in the previous sections and then use that information to prove Theorem 3 . We begin with a theorem that can be thought of as a more detailed version of Theorem 1 .

Theorem 4 Let $G_{p}=G(\Phi)_{p}$ be an adjoint simple algebraic group of type $\Phi$ over $\overline{\mathbb{F}_{p}}$. Let $X \leq G_{p}$ be the connected centralizer of a semisimple element; if $\Phi=B_{n}, C_{n}$ or $D_{n}$ for some $n$ let $q$ be admissible with respect to $X$, otherwise let $q$ be any prime. Then there exists a function $f$ from the set of connected centralizers of semisimple elements of $G_{p}$ to the set of unipotent conjugacy classes of $G_{q}=G(\Phi)_{q}$ such that, for any $u \in f(X), \operatorname{dim} C_{G_{q}}(u)=\operatorname{dim} X$. Furthermore $q^{(X, \Phi)}=o(u)$ unless one of the following holds:

1. $(\Phi, q)=\left(C_{n}, 2\right), X$ has one $C$-factor and $r<l=2^{i}$ for some $i$;

2. $(\Phi, q)=\left(C_{n}, 2\right), X$ has two $C$-factors and $r+1<l=2^{i}$ for some $i$;

3. $(\Phi, q)=\left(B_{n}, 2\right), X$ has $w=0$ and $r=d=2^{i}$ for some $i$;

4. $(\Phi, q)=\left(F_{4}, 3\right)$ and $X=A_{3} A_{1}$;

5. $(\Phi, q)=\left(F_{4}, 2\right)$ and $X=A_{3} T_{1}$;

6. $(\Phi, q)=\left(F_{4}, 5\right)$ and $X=A_{3} T_{1}$;

7. $(\Phi, q)=\left(F_{4}, 2\right)$ and $X=C_{2} T_{2}$;

8. $(\Phi, q)=\left(G_{2}, 2\right)$ and $X=A_{2}$.

In these cases we have $q^{(X, \Phi)}=q . o(u)$. Moreover if $p \neq q$ and $q$ is admissible, then there exists a semisimple element $s \in G_{p}$ such that $C_{G_{p}}(s)^{\circ} \sim_{t} X$ and $o(s)=q^{(X, \Phi)}$.

Proof This is simply a restatement of the conclusions of Theorems 4.1, 5.1, 6.1, 6.2, 7.1, 7.2 and the calculations of Section 2.

We can now use the above result to prove Theorem 3.

Proof of Theorem 3 Note by orders that $s$ is semisimple. Since $s \neq 1$ and $G_{p}$ is adjoint we see that $s \notin Z\left(G_{p}\right)$ and hence $X=C_{G_{p}}(s)^{\circ} \neq G_{p}$. Therefore $o_{\min }(X, \Phi)>1$ and so since $q=o(s) \geq o_{\min }(X, \Phi)$ we must have $q^{(X, \Phi)}=q=o(s)$. Now by definition $q$ is admissible with respect to $X$, so we can apply Theorem 4 to obtain a unipotent conjugacy class in $G_{q}$ whose elements must have order $q$ or 1 . The theorem also gives us that $\operatorname{dim} C_{G_{q}}(u)=\operatorname{dim} X$ and hence $\operatorname{dim} u^{G_{q}}=\operatorname{dim} s^{G_{p}}$. It only remains to show that $o(u)=o(s)$. Here we recall that $o(s)=q^{(X, \Phi)}$ and resort to case analysis using Theorem 4 . The theorem tells us that we can only have $o(u) \neq q^{(X, \Phi)}$ under certain restrictions on $q$ and $X$. To show that we are not in either of the first two cases of the theorem, we observe that if $o(s)=q=2$ and $\Phi=C_{n}$ then when forming $X$ using Algorithm 2.2 we must have deleted one vertex of weight 2 or two vertices of weight 1 from the extended Dynkin diagram. Thus our $X$ would be of the form $C_{n-a} C_{a}$ or $A_{n-1} T_{1}$, but the first two exceptions in Theorem 4 require a single $C$-factor, or two $C$-factors and a central torus of rank $l>r+1 \geq 1$. Therefore we cannot be in those cases. If $o(s)=q=2$ and $\Phi=B_{n}$ then when forming 
$X$ we again must have deleted one vertex of weight 2 or two vertices of weight 1 from the extended Dynkin diagram. Thus our $X$ would be of the form $B_{n-a} D_{a}$ or $B_{n-1} D_{1}$, however both of these have $r=0$ and $d=1$ and the third exception in Theorem 4 requires that $r=d$. Thus we cannot be in that case either. The remaining cases are in type $F_{4}$ or $G_{2}$ and we can see from the tables in Section 2 that the $X \mathrm{~s}$ in these cases cannot be obtained as connected centralizers of elements of order $q$ (since $q \leq o_{\min }(X, \Phi)$ ). The result follows.

\section{References}

1. Carter, R.W.: Finite Groups of Lie Type: Conjugacy Classes and Complex Characters. Wiley Interscience, New York (1985)

2. Hartley, B., Kuzucuoḡu, M.: Centralizers of Elements in Locally Finite Simple Groups. Proc. London Math. Soc. 62, 301-324 (1991)

3. Hesselink, W.H.: Nilpotency in Classical Groups over a Field of Characteristic 2. Math. Z. 166, 165181 (1979)

4. Lawther, R.: Jordan block sizes of unipotent elements in exceptional algebraic groups. Comm. Algebra 23, 4125-4156 (1995)

5. Lawther, R.: Elements of specified order in simple algebraic groups. Trans. Amer. Math. Soc. 357, 221-245 (2004) 